\title{
Non-Periodic Finite-Element Formulation of Orbital-Free Density Functional Theory
}

\author{
Vikram Gavini ${ }^{a}$ Jaroslaw Knap ${ }^{\mathrm{b}}$ Kaushik Bhattacharya ${ }^{\mathrm{a}}$ \\ Michael Ortiz ${ }^{a, *}$ \\ ${ }^{\mathrm{a}}$ Division of Engineering and Applied Science, California Institute of Technology, CA \\ 91125, USA \\ ${ }^{\mathrm{b}}$ Lawrence Livermore National Laboratory, Livermore, CA 94550, USA
}

\begin{abstract}
We propose an approach to perform orbital-free density functional theory calculations in a non-periodic setting using the finite-element method. We consider this a step towards constructing a seamless multi-scale approach for studying defects like vacancies, dislocations and cracks that require quantum mechanical resolution at the core and are sensitive to long range continuum stresses. In this paper, we describe a local real space variational formulation for orbital-free density functional theory, including the electrostatic terms and prove existence results. We prove the convergence of the finite-element approximation including numerical quadratures for our variational formulation. Finally, we demonstrate our method using examples.
\end{abstract}

Key words: Finite Elements, Density functional theory (DFT), Variational Calculus, $\Gamma$-convergence

*Corresponding Author (ortiz@aero.caltech.edu) 


\section{Introduction}

Quantum mechanical calculations have yielded enormous insights into the mechanics and physics of solids in the recent years. They are attractive since they are in principle $a b$ initio and require no empirical input. However, they are extremely expensive, and this expense has limited the size of calculations one can perform. Schrödinger's equation is prohibitively expensive to solve except for a few electron system, and various approaches have been developed to overcome this complexity. Most popular amongst them is the density functional theory based on the work of Hohenberg \& Kohn (1964); Kohn \& Sham (1965). This provides a rigorous reformulation of Schrödinger equation of an N-electron system into a problem of estimating the wave functions and corresponding energies of an effective single electron system. While this approach is exact, it is stated in terms of an unknown exchange and correlation functional and requires an expensive evaluation of the kinetic energy functional. While one can evaluate the exchange and correlation functionals self-consistently, this is expensive and it is common to use an approximate formulation. Similarly it is common to use an approximation for the kinetic energy and this is often referred to as orbital-free density functional theory. We refer the reader to Finnis (2003); Parr \& Yang (1989) for a detailed description and discussion.

Irrespective of whether one is using the Kohn-Sham or the orbital-free approach, one would have to solve the equations in a suitable basis. The plane-wave basis is the most popular, and it lends itself to a computation of the electrostatic interactions naturally using Fourier transforms. However, the plane-wave basis has some very notable disadvantages. Most importantly, it requires periodic boundary conditions and this is not appropriate for various problems of interest in materials science, especially defects. Therefore it is common to artificially consider periodic arrays of defects. Second, a plane-wave basis requires the evaluation of Fourier transforms which affect the scalability of parallel computation. 
Third, the plane-wave basis functions are non-local in the real space, thus resulting in a dense matrix which limits the effectiveness of iterative solutions. This in turn makes it very tricky to embed this in multi-scale approaches which often use real-space formulations to deal with realistic boundary conditions. Although plane-wave basis has been the preferred choice in this area, recently there have been efforts at performing density functional calculations using a finite-element basis in a periodic setting (Pask et al., 1999). Other real-space approaches include GAUSSIAN (Hehre et al., 1969), FPLMTO (Wills \& Cooper, 1987), SIESTA (Soler et al., 2002), ONETEP (Skylaris et al., 2005) and CONQUEST (Bowler et al., 2006) based on specific orbital ansatz or tight-binding.

In this paper, we provide a real-space formulation for orbital-free density functional theory and develop a finite-element method for computing this formulation. In the body of the paper, we confine ourselves to the Thomas-Fermi-Weizsacker kinetic energy functional (Parr \& Yang, 1989; Thomas, 1927; Fermi, 1927) for clarity. However, we show in the Appendix how our approach can be extended to the more recent and accurate kernel kinetic energy functionals (Wang et al., 1998, 1999; Smargiassi \& Madden, 1994; Wang $\&$ Teter, 1992).

An important difficulty in using a real-space formulation is that electrostatic interactions are extended in real-space. So we reformulate the electrostatics as a local variational principle. This converts the problem of computing the ground state energy to a saddle-point variational problem with a local functional in real space. We show that this problem is mathematically well-posed by proving existence of solutions (Theorem 5).

Since our formulation is local and variational, it is natural to discretize it using the finiteelement method. In doing so, we exploit an advantage of the saddle-point formulation and use the same mesh to resolve both the electron density and the electrostatic potential. We prove the convergence of the finite-element approximation, including numerical 
quadratures, using the mathematical technique of $\Gamma$-convergence. This is a notion of convergence of functionals introduced by De Giorgi \& Franzoni (1975) (also see Dal Maso (1993) for a detailed introduction) that has recently been used in a variety of multi-scale problems. In our context, consider a sequence of finer and finer finite-element approximations. These generate a sequence of functionals, and we show that this sequence of functionals $\Gamma$-converge to the exact functional associated with our real-space formulation. While the exact definition is technical, $\Gamma$ - convergence states in spirit that solutions of the sequence of approximate functionals converge to the solution of the exact functional.

Having proved the convergence of our finite-element method, we turn to its numerical implementation. This requires care since the electron densities and electrostatic potential are localized near the atomic cores and are convected as the atomic positions change. Consequently a fixed spatial mesh would be extremely inefficient as we alternate between relaxing the electron density and atomic positions. Therefore, we design a mesh which convects with the atomic position and obtain efficient convergence.

We demonstrate our approach using three sets of examples. The first set of examples are atoms. We begin with a hydrogen atom for which an analytic solution of Schrödinger's equation is known, and also consider other heavier atoms. The second set of examples are nitrogen and carbon-monoxide molecules, for which there are numerous careful calculations. Our results show reasonable agreement for binding energies with experiments and other calculations; however the computed bond lengths are rather poor. These errors are the well-recognized consequence of the use of orbital-free kinetic energy functional in these covalent dimers, rather than our formulation and numerical method. The third set of examples is a series of aluminum clusters ranging from 1 unit (face-centered-cubic) cell to $9 \times 9 \times 9$ unit cells (3730 atoms), and these demonstrate the efficacy and advantages of our approach. Being clusters, they possess no natural periodicity and thus are not 
amenable to plane-wave basis. Second, since the boundaries of the clusters satisfy physically meaningful boundary conditions, it is possible to extract information regarding the scaling of the ground state energy with size. Third, the finite-element method allows one to use unstructured discretization concentrating numerical effort in regions where and only it is necessary with ease and little loss of accuracy. Further, it allows us to adapt the discretization to each atomic position.

This framework is developed with a larger goal in mind, which is to coarse-grain density functional theory in a seamless atomistic-continuum formulation. Such a formulation is necessary to accurately study defects in solids like vacancies, dislocations and cracks where the local structure and long range elastic fields interact in a non-trivial manner. We believe that a local, variational, real-space formulation is a step towards that goal. A further goal is to extend our approach to the exact Kohn-Sham density functional theory setting. These are the subject of current research.

The remainder of the paper is organized as follows. Section 2 describes the formulation. Section 3 collects the important mathematical properties of this functional. Sections 4 and 5 discuss the convergence of the finite-element approximation. Section 6 describes the implementation and Section 7 the examples. We conclude in Section 8 with a short discussion. We have tried to keep the sections on the mathematical analysis and the numerical implementation self-contained so that a reader interested in the former can focus on Sections 3-5 while a reader interested in the latter can focus on Sections 6 and 7. 


\section{Formulation}

The ground state energy in density functional theory is given by (cf, e. g., Finnis (2003); Parr \& Yang (1989))

$$
E(\rho, \mathbf{R})=T_{s}(\rho)+E_{x c}(\rho)+E_{H}(\rho)+E_{e x t}(\rho, \mathbf{R})+E_{z z}(\mathbf{R})
$$

where $\rho$ is the electron density, $\mathbf{R}=\left\{\mathbf{R}_{1}, \ldots, \mathbf{R}_{M}\right\}$ collects the nuclear positions in the system and the different terms are explained presently.

$T_{s}$ is the kinetic energy of non-interacting electrons. A common choice of this is the Thomas-Fermi-Weizsacker family of functionals, which have the form

$$
T_{s}(\rho)=C_{F} \int_{\Omega} \rho^{5 / 3}(\mathbf{r}) d \mathbf{r}+\frac{\lambda}{8} \int_{\Omega} \frac{|\nabla \rho(\mathbf{r})|^{2}}{\rho(\mathbf{r})} d \mathbf{r}
$$

where $C_{F}=\frac{3}{10}\left(3 \pi^{2}\right)^{2 / 3}, \lambda$ is a parameter and $\Omega$ contains the support of $\rho$ (crudely the region where $\rho$ is non-zero). Different values of $\lambda$ are found to work better in different cases (Parr \& Yang, 1989). $\lambda=1$ is the Weizsacker correction and is suitable for rapidly varying electron densities, $\lambda=1 / 9$ gives the conventional gradient approximation and is suitable for slowly varying electron densities, $\lambda=1 / 6$ effectively includes the 4 th order effects and $\lambda=0.186$ was determined from analysis of large atomic-number limit of atoms. This class of functionals makes computations of large and complex systems tractable, though it does have limitations and improvements have been proposed (Wang et al., 1998, 1999; Smargiassi \& Madden, 1994; Wang \& Teter, 1992). We confine our attention to the Thomas-Fermi-Weizsacker family of functionals (2) for now for clarity. However, we explain in the Appendix that our approach can be extended to include the improved functionals.

$E_{x c}$ is the exchange-correlation energy. We use the Local Density Approximation (LDA) 
(Ceperley \& Alder, 1980; Perdew \& Zunger, 1981) given by

$$
E_{x c}(\rho)=\int_{\Omega} \epsilon_{x c}(\rho(\mathbf{r})) \rho(\mathbf{r}) d \mathbf{r}
$$

where $\epsilon_{x c}=\epsilon_{x}+\epsilon_{c}$ is the exchange and correlation energy per electron given by,

$$
\begin{gathered}
\epsilon_{x}(\rho)=-\frac{3}{4}\left(\frac{3}{\pi}\right)^{1 / 3} \rho^{1 / 3} \\
\epsilon_{c}(\rho)= \begin{cases}\frac{\gamma}{1+\beta_{1} \sqrt{r_{s}}+\beta_{2} r_{s}} & r_{s} \geq 1 \\
A \log r_{s}+B+C r_{s} \log r_{s}+D r_{s} \quad r_{s}<1\end{cases}
\end{gathered}
$$

where $r_{s}=\left(\frac{3}{4 \pi \rho}\right)^{1 / 3}$. The values of the constants are different depending on whether the medium is polarized or unpolarized. The values of the constants are $\gamma_{u}=-0.1471$, $\beta_{1_{u}}=1.1581, \beta_{2 u}=0.3446, A_{u}=0.0311, B_{u}=-0.048, C_{u}=0.0014, D_{u}=-0.0108$, $\gamma_{p}=-0.079, \beta_{1 p}=1.2520, \beta_{2 p}=0.2567, A_{p}=0.01555, B_{p}=-0.0269, C_{p}=0.0001$, $D_{p}=-0.0046$.

The last three terms in the functional (1) are electrostatic:

$$
\begin{aligned}
E_{H}(\rho) & =\frac{1}{2} \int_{\Omega} \int_{\Omega} \frac{\rho(\mathbf{r}) \rho\left(\mathbf{r}^{\prime}\right)}{\left|\mathbf{r}-\mathbf{r}^{\prime}\right|} d \mathbf{r} d \mathbf{r}^{\prime}, \\
E_{e x t}(\rho, \mathbf{R}) & =\int_{\Omega} \rho(\mathbf{r}) V_{e x t}(\mathbf{r}) d \mathbf{r} \\
E_{z z}(\mathbf{R}) & =\frac{1}{2} \sum_{I=1}^{M} \sum_{\substack{J \neq 1 \\
J \neq I}}^{M} \frac{Z_{I} Z_{J}}{\left|\mathbf{R}_{I}-\mathbf{R}_{J}\right|} .
\end{aligned}
$$

$E_{H}$ is the classical electrostatic interaction energy of the electron density also referred to as Hartree energy, $E_{\text {ext }}$ is the interaction energy with external field, $V_{\text {ext }}$, induced by nuclear charges and $E_{z z}$ denotes the repulsive energy between nuclei.

The energy functional (1) is local except for two terms: the electrostatic interaction energy of the electrons and the repulsive energy of the nuclei. For this reason, evaluation of the electrostatic interaction energy is the most computationally intensive part of the calculation of the energy functional. Therefore, we seek to write it in a local form. To this end, 
we first regularize the point nuclear charge $Z_{I}$ at $\mathbf{R}_{I}$ with a smooth function $Z_{I} \delta_{\mathbf{R}_{I}}(\mathbf{r})$ which has support in a small ball around $\mathbf{R}_{I}$ and total charge $Z_{I}$. We then rewrite the nuclear energy as

$$
E_{z z}(\mathbf{R})=\frac{1}{2} \int_{\Omega} \int_{\Omega} \frac{b(\mathbf{r}) b\left(\mathbf{r}^{\prime}\right)}{\left|\mathbf{r}-\mathbf{r}^{\prime}\right|} d \mathbf{r} d \mathbf{r}^{\prime}
$$

where $b(\mathbf{r})=\sum_{I=1}^{M} Z_{I} \delta_{\mathbf{R}_{I}}(\mathbf{r})$. Notice that this differs from the earlier formulation by the self-energy of the nuclei, but this is an inconsequential constant depending only on the nuclear charges. Second, we replace the direct Coulomb formula for evaluating the electrostatic energies with the following identity

$$
\begin{aligned}
& \frac{1}{2} \int_{\Omega} \int_{\Omega} \frac{\rho(\mathbf{r}) \rho\left(\mathbf{r}^{\prime}\right)}{\left|\mathbf{r}-\mathbf{r}^{\prime}\right|} d \mathbf{r} d \mathbf{r}^{\prime}+\int_{\Omega} \rho(\mathbf{r}) V_{e x t}(\mathbf{r}) d \mathbf{r}+\frac{1}{2} \int_{\Omega} \int_{\Omega} \frac{b(\mathbf{r}) b\left(\mathbf{r}^{\prime}\right)}{\left|\mathbf{r}-\mathbf{r}^{\prime}\right|} d \mathbf{r} d \mathbf{r}^{\prime} \\
& =-\inf _{\phi \in H^{1}\left(\mathbb{R}^{3}\right)}\left\{\frac{1}{8 \pi} \int_{\mathbb{R}^{3}}|\nabla \phi(\mathbf{r})|^{2} d \mathbf{r}-\int_{\mathbb{R}^{3}}(\rho(\mathbf{r})+b(\mathbf{r})) \phi(\mathbf{r}) d \mathbf{r}\right\}
\end{aligned}
$$

where we assume that $\rho \in H^{-1}\left(\mathbb{R}^{3}\right)$. Briefly, note that the Euler-Lagrange equation associated with the variational problem above is

$$
\frac{-1}{4 \pi} \Delta \phi=\rho+b
$$

These have an unique solution

$$
\phi(\mathbf{r})=\int_{\Omega} \frac{\rho\left(\mathbf{r}^{\prime}\right)}{\left|\mathbf{r}-\mathbf{r}^{\prime}\right|} d \mathbf{r}^{\prime}+\int_{\Omega} \frac{b\left(\mathbf{r}^{\prime}\right)}{\left|\mathbf{r}-\mathbf{r}^{\prime}\right|} d \mathbf{r}^{\prime}=\int_{\Omega} \frac{\rho\left(\mathbf{r}^{\prime}\right)}{\left|\mathbf{r}-\mathbf{r}^{\prime}\right|} d \mathbf{r}^{\prime}+V_{e x t} .
$$

Substituting this into the variational problem and integrating by parts gives us the desired identity.

This identity (10) allows us to write the energy functional in the local form,

$$
E(\rho, \mathbf{R})=\sup _{\phi \in H^{1}\left(\mathbb{R}^{3}\right)} L(\rho, \mathbf{R}, \phi)
$$

where we introduce the Lagrangian

$$
\begin{aligned}
L(\rho, \mathbf{R}, \phi) & =C_{F} \int_{\Omega} \rho^{5 / 3}(\mathbf{r}) d \mathbf{r}+\frac{\lambda}{8} \int_{\Omega} \frac{|\nabla \rho(\mathbf{r})|^{2}}{\rho(\mathbf{r})} d \mathbf{r}+\int_{\Omega} \epsilon_{x c}(\rho(\mathbf{r})) \rho(\mathbf{r}) d \mathbf{r} \\
& -\frac{1}{8 \pi} \int_{\mathbb{R}^{3}}|\nabla \phi(\mathbf{r})|^{2} d \mathbf{r}+\int_{\mathbb{R}^{3}}(\rho(\mathbf{r})+b(\mathbf{r})) \phi(\mathbf{r}) d \mathbf{r} .
\end{aligned}
$$


The problem of determining the ground-state electron density and the equilibrium positions of the nuclei can now be expressed as the minimum problem

$$
\begin{array}{r}
\inf _{\rho \in H_{0}^{-1}(\Omega),} \in\left(\rho \in \mathbb{R}^{3 M}\right. \\
\rho(\mathbf{r}) \geq 0 \\
\text { subject to: } \quad \begin{array}{r}
\mathbf{R}) \\
\int_{\Omega} \rho(\mathbf{r}) d \mathbf{r}=N,
\end{array}
\end{array}
$$

where $N$ is the number of electrons of the system. Equivalently, the problem can be formulated in the saddle-point form

$$
\inf _{\rho \in H_{0}^{-1}(\Omega), \mathbf{R} \in \mathbb{R}^{3 M}} \sup _{\phi \in H^{1}\left(\mathbb{R}^{3}\right)} L(\rho, \mathbf{R}, \phi)
$$

subject to:

$$
\begin{aligned}
\rho(\mathbf{r}) & \geq 0 \\
\int_{\Omega} \rho(\mathbf{r}) d \mathbf{r} & =N .
\end{aligned}
$$

The constraint of $\rho \geq 0$ can be imposed by making the substitution

$$
\rho=u^{2}
$$

which results in the Lagrangian

$$
\begin{aligned}
L(u, \mathbf{R}, \phi) & =C_{F} \int_{\Omega} u^{10 / 3}(\mathbf{r}) d \mathbf{r}+\frac{\lambda}{2} \int_{\Omega}|\nabla u(\mathbf{r})|^{2} d \mathbf{r}+\int_{\Omega} \varepsilon_{x c}\left(u^{2}(\mathbf{r})\right) u^{2}(\mathbf{r}) d \mathbf{r} \\
& -\frac{1}{8 \pi} \int_{\mathbb{R}^{3}}|\nabla \phi(\mathbf{r})|^{2} d \mathbf{r}+\int_{\mathbb{R}^{3}}\left(u^{2}(\mathbf{r})+b(\mathbf{r})\right) \phi(\mathbf{r}) d \mathbf{r}
\end{aligned}
$$

and the energy

$$
E(u, \mathbf{R})=\sup _{\phi \in H^{1}\left(\mathbb{R}^{3}\right)} L(u, \mathbf{R}, \phi) .
$$

With this representation, the minimum problem (15) becomes

$$
\begin{aligned}
& \inf _{u^{2} \in H_{0}^{-1}(\Omega),}, \mathbf{R} \in \mathbb{R}^{3 M} \\
& \text { subject to: } \quad \int_{\Omega} u^{2}(\mathbf{r}) d \mathbf{r}=N
\end{aligned}
$$


and the saddle-point problem (16) becomes

$$
\begin{aligned}
& \inf _{u^{2} \in H_{0}^{-1}(\Omega),} \sup _{\mathbf{R} \in \mathbb{R}^{3 M}} L(u, \mathbf{R}, \phi) \\
& \text { subject to: } \quad \int_{\Omega} u^{2}(\mathbf{R}) d \mathbf{r}=N .
\end{aligned}
$$

The preceding local variational characterization of the ground-state electronic structure constitutes the basis of the finite-element approximation schemes described subsequently.

\section{Properties of the DFT variational problem}

We begin by establishing certain properties of the DFT variational problem that play a fundamental role in the analysis of convergence presented in the sequel. To keep the analysis simple we treat the electrostatics on a large but bounded domain with compact support. To this end, we consider energy functionals $E: W^{1, p}(\Omega) \rightarrow \overline{\mathbb{R}}$ of the form

$$
\begin{array}{r}
E(u)=\int_{\Omega} f(\nabla u) d \mathbf{r}+\int_{\Omega} g(u) d \mathbf{r}+J(u) \\
J(u)=-\inf _{\phi \in H_{0}^{1}(\Omega)}\left\{\frac{1}{2} \int_{\Omega}|\nabla \phi|^{2} d \mathbf{r}-\int_{\Omega}\left(u^{2}+b(\mathbf{r})\right) \phi d \mathbf{r}\right\},
\end{array}
$$

where $\Omega$ is an open bounded subset of $\mathbb{R}^{N}$, with $\partial \Omega$ Lipschitz continuous. $b(\mathbf{r})$ is a smooth, bounded function in $\mathbb{R}^{N}$. We assume:

(i) $f$ is convex and continuous on $\mathbb{R}^{N}$.

(ii) $f$ satisfies the growth condition, $c_{0}|\psi|^{p}-a_{0} \leq f(\psi) \leq c_{1}|\psi|^{p}-a_{1}, 1<p<\infty$, where $c_{0}, c_{1} \in \mathbb{R}^{+}, a_{0}, a_{1} \in \mathbb{R}$.

(iii) $g$ is continuous on $\mathbb{R}$.

(iv) $g$ satisfies the growth condition, $c_{2}|s|^{q}-a_{2} \leq g(s) \leq c_{3}|s|^{q}-a_{3}, q \geq p$, where $c_{2}, c_{3} \in \mathbb{R}^{+}$, $a_{2}, a_{3} \in \mathbb{R}$.

Let $F: W^{1, p}(\Omega) \rightarrow \overline{\mathbb{R}}$ and $G: W^{1, p}(\Omega) \rightarrow \overline{\mathbb{R}}$ be functionals defined by, 


$$
F(u)=\int_{\Omega} f(\nabla u) d \mathbf{r} \quad G(u)=\int_{\Omega} g(u) d \mathbf{r} .
$$

We note that the growth conditions imply, $|f(\psi)| \leq c\left(1+|\psi|^{p}\right)$ and $|g(s)| \leq c\left(1+|s|^{q}\right)$. Hence, it follows that, $F(u)$ is continuous in $W^{1, p}(\Omega)$ and $G(u)$ is continuous in $L^{q}(\Omega)$, cf, e. g., Remark 2.10, Braides (2002).

Let $X=\left\{u \mid u \in W^{1, p}(\Omega),\|u\|_{L^{2}(\Omega)}=1\right\}$ with norm induced from $W^{1, p}(\Omega)$. Let, $\frac{1}{p^{*}}=$ $\frac{1}{p}-\frac{1}{N}$.

Lemma $1 X$ is closed in the weak topology of $W^{1, p}(\Omega)$ if $p^{*}>2$.

Proof. We can rewrite $X$ as $X=W^{1, p}(\Omega) \cap K$, where $K=\left\{u \in L^{2}(\Omega) \mid\|u\|_{L^{2}(\Omega)}=1\right\}$. Let $\left(u_{h}\right) \in X, u_{h} \rightarrow u$ in $W^{1, p}(\Omega)$. If $p^{*}>2$, then $W^{1, p}(\Omega)$ is a compact injection into $L^{2}(\Omega)$. Hence, $u_{h} \rightarrow u$ in $L^{2}(\Omega)$. Thus, $1=\left\|u_{h}\right\|_{L^{2}(\Omega)} \rightarrow\|u\|_{L^{2}(\Omega)}$ Hence, $u \in K$ and it follows that $\mathrm{X}$ is closed in the weak topology of $W^{1, p}(\Omega)$

In this section we establish the existence of a minimum point of the energy functional $E(u)$ in $X$. Let,

$$
I(\phi, u)=\frac{1}{2} \int_{\Omega}|\nabla \phi|^{2} d \mathbf{r}-\int_{\Omega}\left(u^{2}+b\right) \phi d \mathbf{r}, \quad \phi \in H_{0}^{1}(\Omega) \quad u \in W^{1, p}(\Omega) .
$$

Hence,

$$
J(u)=-\inf _{\phi \in H_{0}^{1}(\Omega)} I(\phi, u) .
$$

For every $u \in L^{4}(\Omega), I(., u)$ admits a minimum. This follows from Poincaré inequality and Lax-Milgram Lemma. Therefore,

$$
J(u)=-\min _{\phi \in H_{0}^{1}(\Omega)} I(\phi, u)
$$

Lemma $2 J$ is continuous in $L^{4}(\Omega)$. 
Proof. If $\phi_{u}$ denotes the minimizer of $I(., u)$, then for every $u, v \in L^{4}(\Omega)$, we have,

$$
\int_{\Omega} \nabla\left(\phi_{u}-\phi_{v}\right) \cdot \nabla \psi d \mathbf{r}=\int_{\Omega}\left(u^{2}-v^{2}\right) \psi d \mathbf{r} \quad \forall \psi \in H_{0}^{1}(\Omega) .
$$

Hence, from Poincaré and Cauchy-Schwartz inequality, it is immediate that,

$$
\left\|\phi_{u}-\phi_{v}\right\|_{H_{0}^{1}(\Omega)} \leq C\left\|u^{2}-v^{2}\right\|_{L^{2}(\Omega)}
$$

Continuity of $J$ thus follows.

Let us denote by Hypothesis $H$, the condition, $p^{*}>\max \{q, 4\}$.

Lemma 3 If the Hypothesis $H$ is satisfied, then $E$ is lower semi-continuous (l.s.c) in the weak topology of $X$.

Proof. We noted previously that $F$ is continuous in $W^{1, p}(\Omega)$. As $F$ is convex, it follows that $F$ is l.s.c in the weak topology of $W^{1, p}(\Omega)$ (cf, e. g. Prop. 1.18, Dal Maso (1993)). If the hypothesis $H$ is satisfied, then $W^{1, p}(\Omega)$ is a compact injection into $L^{q}(\Omega)$ and $L^{4}(\Omega)$. $G$ is continuous in $L^{q}(\Omega)$, as noted previously, and from Lemma $2, J$ is continuous in $L^{4}(\Omega)$. Hence, it follows that, $G$ and $J$ are 1.s.c and thus $E$ is 1.s.c in the weak topology of $W^{1, p}(\Omega)$. As $X$ is a subset of $W^{1, p}(\Omega)$, it follows that $E$ is l.s.c in the weak topology of $X$.

Lemma $4 E$ is coercive in the weak topology of $X$.

Proof. If we establish the coercivity of $E$ in the weak topology of $W^{1, p}(\Omega)$, the coercivity of $E$ in the weak topology of $X$ follows from Lemma 1 . We note that $J(u) \geq 0$. Hence,

$$
\begin{aligned}
E(u) & \geq c_{0}\|\nabla u\|_{L^{p}(\Omega)}^{p}+c_{2}\|u\|_{L^{q}(\Omega)}^{q}-\left(a_{0}+a_{2}\right) \Omega \\
& \geq c_{0}\|\nabla u\|_{L^{p}(\Omega)}^{p}+\frac{c_{1}}{C_{\Omega}^{q}}\|u\|_{L^{p}(\Omega)}^{q}-C=K(u) \text { as } p \leq q
\end{aligned}
$$


If the function $K$ is bounded, then $\|u\|_{W^{1, p}(\Omega)}$ is bounded. As $W^{1, p}(\Omega)$ is reflexive $(1<$ $p<\infty)$, it follows that $K$ is coercive in the weak topology of $W^{1, p}(\Omega)$. Hence, $E$ is coercive in the weak topology of $W^{1, p}(\Omega)$ and from Lemma 1, $E$ is coercive in the weak topology of $X$.

Theorem $5 E(u)$ has a minimum in $X$.

Proof. It follows from Lemma 3, Lemma 4 and Theorem 1.15, Dal Maso (1993).

The orbital-free density functional under consideration falls into the class of functionals being discussed with $J(u)$ representing the classical electrostatic interaction energy. The constraint on electron density is imposed explicitly through the space $X$. It is easy to check that the energy functional satisfies conditions (i)-(iv) with $p=2, q=10 / 3$. As $\Omega \subset \mathbb{R}^{3}$, we estimate $p^{*}=6$. Hence, the hypothesis $H$ is satisfied and all the results apply to the specific energy functional.

\section{$4 \quad \Gamma$-Convergence of the Finite-Element Approximation}

Finite-element approximations to the solutions of the DFT variational problem are obtained by restricting minimization to a sequence of increasing finite-dimensional subspaces of $X$. Thus, let $T_{h}$ be a sequence of triangulations of $\Omega$ of decreasing mesh size, and let $X_{h}$ be the corresponding sequence of subspaces of $X$ consisting of functions whose restriction to every cell in $T_{h}$ is a polynomial function of degree $k \geq 1$. A standard result in approximation theory (cf, e. g., Ciarlet (2002)) shows that the sequence $\left(X_{h}\right)$ is dense in $X$, i. e., for every $u \in X$ there is a sequence $u_{h} \in X_{h}$ such that $u_{h} \rightarrow u$. Let, $X_{1_{h}}=\left\{\phi \mid \phi \in H_{0}^{1}(\Omega), \phi\right.$ is piece-wise polynomial function corresponding to triangulation $\left.T_{h}\right\}$, denote a sequence of constrained spaces of the space $H_{0}^{1}(\Omega)$. The sequence 
of spaces, $\left(X_{1_{h}}\right)$, is such that $\cup_{h} X_{1_{h}}$ is dense in $H_{0}^{1}(\Omega)$. We now define a sequence of finite-element energy functionals

$$
E_{h}(u)= \begin{cases}F(u)+G(u)+J_{h}(u), & \text { if } u \in X_{h} \\ +\infty, & \text { otherwise }\end{cases}
$$

where

$$
J_{h}(u)=-\min _{\phi \in H_{0}^{1}(\Omega)} I_{h}(\phi, u)
$$

and

$$
I_{h}(\phi, u)= \begin{cases}I(\phi, u), & \text { if } \phi \in X_{1_{h}}, u \in X_{h} ; \\ +\infty, & \text { otherwise; }\end{cases}
$$

Then, we would like to establish convergence of the sequence of functionals $E_{h}$ to $E$ in a sense such that the corresponding convergence of minimizers is guaranteed. This natural notion of convergence of variational problems is provided by $\Gamma$-convergence (cf, e. g., Dal Maso (1993) for comprehensive treatises of the subject). In the remainder of this section, we show the $\Gamma$-convergence of the finite-element approximation and attendant convergence of the minima. We also extend the analysis of convergence to approximations obtained using numerical quadrature.

To analyze the behavior of the sequence of functionals, $E_{h}$, it is important to understand the behavior of $J_{h}$. We first note some properties of $J_{h}$ before analyzing $E_{h}$.

Lemma 6 If $u_{h} \rightarrow u$ in $L^{4}(\Omega)$, then for any $\phi_{h} \rightarrow \phi$ in $H_{0}^{1}(\Omega), \liminf _{h \rightarrow \infty} I\left(\phi_{h}, u_{h}\right) \geq I(\phi, u)$.

Proof. $I(\phi, u)=\frac{1}{2} \int_{\Omega}|\nabla \phi|^{2} d \mathbf{r}-\int_{\Omega}\left(u^{2}+b\right) \phi d \mathbf{r}$. L.s.c of $\int_{\Omega}|\nabla \phi|^{2} d \mathbf{r}$ in the weak topology of $H_{0}^{1}(\Omega)$ follows from Prop 2.1, Dal Maso (1993). As $u_{h} \rightarrow u$ in $L^{4}(\Omega), \lim _{h \rightarrow \infty} \int_{\Omega}\left(u_{h}^{2}+b\right) \phi_{h} d \mathbf{r}=$ $\int_{\Omega}\left(u^{2}+b\right) \phi d \mathbf{r}$. Putting both the terms together, we get, $\liminf _{h \rightarrow \infty} I\left(\phi_{h}, u_{h}\right) \geq I(\phi, u)$. 
Lemma 7 If $u_{h} \rightarrow u$ in $L^{4}(\Omega)$, then $\left(I_{h}\left(., u_{h}\right)\right)$ is equi-coercive in the weak topology of $H_{0}^{1}(\Omega)$

\section{Proof.}

$$
I(\phi, u) \geq C\|\phi\|_{H_{0}^{1}(\Omega)}^{2}-\left(\left\|u^{2}\right\|_{L^{2}(\Omega)}+\|b\|_{L^{2}(\Omega)}\right)\|\phi\|_{L^{2}(\Omega)}
$$

$I_{h}\left(., u_{h}\right) \geq I\left(., u_{h}\right) \geq I^{*}$ where $I^{*}(\phi)=C\|\phi\|_{H_{0}^{1}(\Omega)}^{2}-K\|\phi\|_{L^{2}(\Omega)}, K=\sup _{h}\left\|u_{h}{ }^{2}\right\|_{L^{2}(\Omega)}+$ $\|b\|_{L^{2}(\Omega)}$. Since, $u_{h} \rightarrow u$ in $L^{4}(\Omega)$ and $b$ is a bounded function, $K$ is bounded. This implies, $I^{*}$ is coercive in the weak topology of $H_{0}^{1}(\Omega)$. Thus it follows that, $\left(I_{h}\left(., u_{h}\right)\right)$ is equi-coercive in the weak topology of $H_{0}^{1}(\Omega)$.

Theorem 8 If $\left(u_{h}\right) \in\left(X_{h}\right)$ is a sequence such that $u_{h} \rightarrow u$ in $L^{4}(\Omega)$, then $I_{h}\left(., u_{h}\right) \rightarrow^{\Gamma}$ $I(., u)$ in weak topology of $H_{0}^{1}(\Omega)$.

Proof. Let $\left(\phi_{h}\right)$ be any sequence $\ni \phi_{h} \rightarrow \phi$ in $H_{0}^{1}(\Omega) . I_{h}\left(\phi_{h}, u_{h}\right) \geq I\left(\phi_{h}, u_{h}\right)$. Hence, $\liminf _{h \rightarrow \infty} I_{h}\left(\phi_{h}, u_{h}\right) \geq \liminf _{h \rightarrow \infty} I\left(\phi_{h}, u_{h}\right)$. But from Lemma 6, $\liminf _{h \rightarrow \infty} I\left(\phi_{h}, u_{h}\right) \geq$ $I(\phi, u)$. Hence, $\liminf _{h \rightarrow \infty} I_{h}\left(\phi_{h}, u_{h}\right) \geq I(\phi, u)$. Now we construct the recovery sequence from interpolated functions. Let $\left(\phi_{h}\right)$ be a sequence constructed from the interpolation functions of successive triangulations such that $\phi_{h} \rightarrow \phi$ in $H_{0}^{1}$. As $\phi_{h} \rightarrow \phi$ in $H_{0}^{1}(\Omega),\left\|\nabla \phi_{h}\right\|_{L^{2}(\Omega)} \rightarrow\|\nabla \phi\|_{L^{2}(\Omega)}$. Also, as $u_{h} \rightarrow u$ in $L^{4}(\Omega), \lim _{h \rightarrow \infty} \int_{\Omega}\left(u_{h}^{2}+b\right) \phi_{h} d \mathbf{r}=$ $\int_{\Omega}\left(u^{2}+b\right) \phi d \mathbf{r}$. Hence, $\lim _{h \rightarrow \infty} I_{h}\left(\phi_{h}, u_{h}\right)=I(\phi, u)$. This shows that, $I_{h}\left(., u_{h}\right) \rightarrow^{\Gamma}$ $I(., u)$ in weak topology of $H_{0}^{1}(\Omega)$.

Theorem 9 If $\left(u_{h}\right) \in\left(X_{h}\right)$ is a sequence such that $u_{h} \rightarrow u$ in $L^{4}(\Omega)$, then $\lim _{h \rightarrow \infty} J_{h}\left(u_{h}\right)=$ $J(u)$.

Proof. Follows from Lemma 7, Theorem 8 and Theorem 7.8, Dal Maso (1993). 
Lemma 10 Let $u_{h} \rightarrow u$ in $X$, then $\liminf _{h \rightarrow \infty} E_{h}\left(u_{h}\right) \geq E(u)$ if the hypothesis $H$ is satisfied.

Proof. We need to consider 2 cases.

Case 1: There is no sub-sequence $\left(u_{h_{k}}\right)$ such that $\left(u_{h_{k}}\right) \in X_{h_{k}}$

$\liminf _{h \rightarrow \infty} E_{h}\left(u_{h}\right)=+\infty$. Hence, $\liminf _{h \rightarrow \infty} E_{h}\left(u_{h}\right) \geq E(u)$.

Case $2: \exists$ sub-sequence $\left(u_{h_{k}}\right)$ such that $\left(u_{h_{k}}\right) \in X_{h_{k}}$

Using Theorem 9, the proof for this case follows on the same lines as Lemma 3.

Theorem $11 E_{h} \rightarrow^{\Gamma} E$ in weak topology of $X$ if the hypothesis $H$ is satisfied.

Proof. Let $\left(u_{h}\right)$ be any sequence $\ni u_{h} \rightarrow u$ in $X$. From Lemma 10, it follows that $\liminf _{h \rightarrow \infty} E_{h}\left(u_{h}\right) \geq E(u)$.

Now lets construct the recovery sequence. Let $\left(u_{h}\right)$ be a sequence constructed from the interpolation functions of successive triangulations such that, $u_{h} \rightarrow u$ in $X$. From Theorem 9 and continuity of $F$ and $G$, it follows that $\lim _{h \rightarrow \infty} E_{h}\left(u_{h}\right)=E(u)$. Thus, $E_{h} \rightarrow^{\Gamma} E$ in weak topology of $X$.

Lemma $12\left(E_{h}\right)$ is equi-coercive in the weak topology of $X$ if the hypothesis $H$ is satisfied.

Proof. Noting that $E_{h}(u) \geq F(u)+G(u)+J_{h}(u)$ and $J_{h}(u) \geq 0$ if $u \in X_{h}$, the proof follows on the same lines as Lemma 4.

Theorem $13 \lim _{h \rightarrow \infty} \inf _{X} E_{h}=\min _{X} E$ if the hypothesis $H$ is satisfied.

Proof. Follows from Lemma 12, Theorem 11 and Theorem 7.8, Dal Maso (1993). 


\section{5 -convergence of the Finite-Element Approximation with Numerical Quadra- tures}

Let $f: \Omega \rightarrow \mathbb{R}, \Omega \subset \mathbb{R}^{N}, \Omega$ bounded, be a function in $W^{n+1,1}(\Omega)$ and $I=\int_{\Omega} f(\mathbf{r}) d \mathbf{r}$. Define the quadrature of $I$ to be,

$$
\tilde{I}=\sum_{i=1}^{P} C_{i} f\left(\mathbf{r}\left(\xi_{i}\right)\right)
$$

where, $P$ denotes the number of quadrature points and $C$ and $\xi$ denote the weights and quadrature points. If the quadrature rule is of $n^{\text {th }}$ order, then the values of $C$ and $\xi$ are determined such that all polynomials upto degree $n$ are integrated exactly. If the quadrature rule is $n^{\text {th }}$ order, then the error due to the quadrature rule is given by

$$
|\tilde{I}-I| \leq K C_{\Omega}^{(n+1)} \int_{\Omega}\left|f^{(n+1)}(\mathbf{r})\right| d \mathbf{r},
$$

where $f^{(n+1)}$ denotes the $n+1^{\text {th }}$ derivative of $f$ and $C_{\Omega}$ represents the size of the domain. Define $\tilde{I}_{h}$ as,

$$
\tilde{I}_{h}(\phi, u)= \begin{cases}\tilde{I}(\phi, u), & \text { if } \phi \in X_{1_{h}}, u \in X_{h} \\ +\infty, & \text { otherwise; }\end{cases}
$$

We rewrite $\tilde{I}_{h}$ as

$$
\tilde{I}_{h}(\phi, u)=I_{h}(\phi, u)+\Delta I_{h}(\phi, u)
$$

where $\Delta I_{h}(\phi, u)$ is a perturbation of $I_{h}(\phi, u)$ introduced due to numerical quadrature and is given by

$$
\Delta I_{h}(\phi, u)= \begin{cases}\tilde{I}(\phi, u)-I(\phi, u), & \text { if } \phi \in X_{1_{h}}, u \in X_{h} \\ 0, & \text { otherwise; }\end{cases}
$$

To estimate the error in the energy introduced due to the quadrature, we assume that the family of triangulations $\left(T_{h}\right)$ are regular, affine and satisfy the inverse assumption (cf, e. g., Ciarlet (2002)). If the quadrature rule is $n^{\text {th }}$ order, then the error due to the quadrature for $\phi \in X_{1_{h}}$ and $u \in X_{h}$ is given by 


$$
\begin{aligned}
\left|\Delta I_{h}(\phi, u)\right| & \leq C h_{0}^{n+1} \sum_{i} \int_{e_{i}}\left|D^{n+1}\left[\frac{1}{2}|\nabla \phi|^{2}-\left(u^{2}+b\right) \phi\right]\right| d \mathbf{r} \\
& \leq C h_{0}^{n+1} \sum_{i} \int_{e_{i}}\left\{\left.\left|D^{n+1}\right| \nabla \phi\right|^{2}|+| D^{n+1}\left(\left(u^{2}+b\right) \phi\right) \mid\right\} d \mathbf{r} \\
& \leq C h_{0}^{n+1} \sum_{i} \int_{e_{i}}\left\{\left.\left|D^{n+1}\right| \nabla \phi\right|^{2}\left|+C_{1} h_{0}^{-n}\right| D\left(u^{2} \phi\right)\left|+C_{2} h_{0}^{-n}\right| D(\phi) \mid\right\} d \mathbf{r}
\end{aligned}
$$

where $e_{i}$ denotes the $i^{t h}$ element and $h_{0}$ is characteristic of the size of the largest element in the finite-element mesh. The last inequality in (23) is obtained by using the inverse inequality (Ciarlet, 2002). We note that, as $h \rightarrow \infty, h_{0} \rightarrow 0$. Let $k$ denote the degree of polynomials used for finite-element interpolation.

Lemma 14 If $\left(u_{h}\right) \in\left(X_{h}\right)$ is a sequence such that $u_{h} \rightarrow u$ in $X,(n-2 k+3)>0, p \geq 2$ and the hypothesis $H$ is satisfied, then $\left(\Delta I_{h}\left(., u_{h}\right)\right)$ is continuously convergent to the zero function in $H_{0}^{1}(\Omega)$.

Proof. If $\phi \notin X_{1_{h}}$, then by definition, $\Delta I_{h}\left(\phi, u_{h}\right)=0$. Hence, we need to consider only the case where $\phi \in X_{1_{h}}$. If $\phi \in X_{1_{h}}$, then from (23),

$$
\left|\Delta I_{h}\left(\phi, u_{h}\right)\right| \leq C h_{0}^{n+1} \sum_{i} \int_{e_{i}}\left\{\left.\left|D^{n+1}\right| \nabla \phi\right|^{2}\left|+C_{1} h_{0}^{-n}\right| D\left(u_{h}^{2} \phi\right)\left|+C_{2} h_{0}^{-n}\right| D(\phi) \mid\right\} d \mathbf{r}
$$

If $(n-2 k+3)>0$, then $D^{n+1}|\nabla \phi|^{2}=0$. Hence,

$$
\begin{aligned}
\left|\Delta I_{h}\left(\phi, u_{h}\right)\right| & \leq C h_{0} \sum_{i} \int_{e_{i}}\left|D\left(u_{h}^{2} \phi\right)\right| d \mathbf{r}+C_{1} h_{0} \sum_{i} \int_{e_{i}}|D(\phi)| d \mathbf{r} \\
& \leq C h_{0}\left\{\left\|\nabla u_{h}\right\|_{L^{2}(\Omega)}\left\|u_{h} \phi\right\|_{L^{2}(\Omega)}+\|\nabla \phi\|_{L^{2}(\Omega)}\left\|u_{h}\right\|_{L^{4}(\Omega)}^{2}\right\}+C_{1} h_{0}\|\nabla \phi\|_{L^{1}(\Omega)} \\
& \leq C h_{0}\left\{\left\|\nabla u_{h}\right\|_{L^{2}(\Omega)}\left\|u_{h}\right\|_{L^{4}(\Omega)}\|\phi\|_{L^{4}(\Omega)}+\|\nabla \phi\|_{L^{2}(\Omega)}\left(\left\|u_{h}\right\|_{L^{4}(\Omega)}^{2}+C_{2}\right)\right\} .
\end{aligned}
$$

As the hypothesis $H$ is satisfied, $H_{0}^{1}(\Omega)$ and $W^{1, p}(\Omega)$ are compact injections into $L^{4}(\Omega)$ and all the norms make sense. As, $u_{h} \rightarrow u$ in $X$, it follows that norms $\left\|\nabla u_{h}\right\|_{L^{2}(\Omega)}$ and $\left\|u_{h}\right\|_{L^{4}(\Omega)}$ are uniformly bounded. Hence, it follows that $\left(\Delta I_{h}\left(., u_{h}\right)\right)$ is continuously 
convergent to the zero function.

Theorem 15 If $\left(u_{h}\right) \in\left(X_{h}\right)$ is a sequence such that $u_{h} \rightarrow u$ in $X,(n-2 k+3)>0, p \geq 2$ and the hypothesis $H$ is satisfied, then $\tilde{I}_{h}\left(., u_{h}\right) \rightarrow^{\Gamma} I(., u)$ in weak topology of $H_{0}^{1}(\Omega)$.

Proof. $\tilde{I}_{h}\left(., u_{h}\right)=I_{h}\left(., u_{h}\right)+\Delta I_{h}\left(., u_{h}\right)$. From Lemma 14, it follows that $\left(\Delta I_{h}\left(., u_{h}\right)\right)$ is continuously convergent to zero. Hence, from Prop. 6.20, Dal Maso (1993), it follows that $\tilde{I}_{h}\left(., u_{h}\right) \rightarrow^{\Gamma} I(., u)$ in weak topology of $H_{0}^{1}(\Omega)$.

Theorem 16 If $\left(u_{h}\right) \in\left(X_{h}\right)$ is a sequence such that $u_{h} \rightarrow u$ in $X,(n-2 k+3)>$ $0, p \geq 2, N<4$ and the hypothesis $H$ is satisfied, then $\lim _{h \rightarrow \infty} \inf _{H_{0}^{1}(\Omega)} \tilde{I}_{h}\left(., u_{h}\right)=$ $\min _{H_{0}^{1}(\Omega)} I(., u)$, i.e. $\lim _{h \rightarrow \infty} \tilde{J}_{h}\left(u_{h}\right)=J(u)$.

Proof. To show this we need to show that $\tilde{I}_{h}$ is equi-coercive in the weak topology of $H_{0}^{1}(\Omega)$. For $\phi \in X_{1_{h}}$, from (22) and (24),

$$
\begin{aligned}
\tilde{I}_{h}\left(\phi, u_{h}\right) & \geq I_{h}\left(\phi, u_{h}\right)-C h_{0}\left\{\left\|\nabla u_{h}\right\|_{L^{2}(\Omega)}\left\|u_{h}\right\|_{L^{4}(\Omega)}\|\phi\|_{L^{4}(\Omega)}+\|\nabla \phi\|_{L^{2}(\Omega)}\left(\left\|u_{h}\right\|_{L^{4}(\Omega)}^{2}+C_{2}\right)\right\} \\
& \geq C_{1}\|\phi\|_{H_{0}^{1}(\Omega)}^{2}-C_{2}\|\phi\|_{L^{2}(\Omega)}-C_{3} h_{0}\|\nabla \phi\|_{L^{2}(\Omega)}-C_{4} h_{0}\|\phi\|_{L^{4}(\Omega)}
\end{aligned}
$$

Using Inverse Inequality, $\|\phi\|_{L^{4}(\Omega)} \leq C h_{0}^{-N / 4}\|\phi\|_{L^{2}(\Omega)}$. Hence, we have,

$\tilde{I}_{h}\left(\phi, u_{h}\right) \geq C_{1}\|\phi\|_{H_{0}^{1}(\Omega)}^{2}-C_{2}\|\phi\|_{L^{2}(\Omega)}-C_{3} h_{0}\|\nabla \phi\|_{L^{2}(\Omega)}-C h_{0}^{1-N / 4}\|\phi\|_{L^{2}(\Omega)} \quad\left(C_{1}>0\right)$

If $\phi \notin X_{1_{h}}$, then $\tilde{I}_{h}\left(\phi, u_{h}\right)=\infty$. Hence, for any $\phi$ we have,

$\tilde{I}_{h}\left(\phi, u_{h}\right) \geq C_{1}\|\phi\|_{H_{0}^{1}(\Omega)}^{2}-C_{2}\|\phi\|_{L^{2}(\Omega)}-C_{3} h_{0}\|\nabla \phi\|_{L^{2}(\Omega)}-C h_{0}^{1-N / 4}\|\phi\|_{L^{2}(\Omega)} \quad\left(C_{1}>0\right)$

As all the terms appearing with a negative sign are lower order, it follows that $\tilde{I}_{h}$ is equicoercive in the weak topology of $H_{0}^{1}(\Omega)$. Hence, the result follows from Theorem 15 and Theorem 7.8, Dal Maso (1993). 
Returning to the energy functional, lets define,

$$
\tilde{E}_{h}(u)= \begin{cases}\tilde{F}(u)+\tilde{G}(u)+\tilde{J}_{h}(u), & \text { if } u \in X_{h} \\ +\infty, & \text { otherwise }\end{cases}
$$

If $f$ is a polynomial function of degree $d$ which satisfies the condition $n-d(k-1) \geq 0$ and $g^{\prime}(u) \in L^{2}(\Omega)$, then for $u \in X_{h}$, we have the error estimate for a quadrature of $n^{t h}$ order as,

$$
\left|\tilde{E}_{h}(u)-E_{h}(u)\right| \leq C h_{0}^{n+1} \sum_{i} \int_{e_{i}}\left|D^{n+1}[f(\nabla u)+g(u)]\right| d \mathbf{r}+\left|\tilde{J}_{h}(u)-J_{h}(u)\right|
$$

If $f$ is a polynomial function of degree $d$ which satisfies the condition $n-d(k-1) \geq 0$, then $D^{n+1}(f(\nabla u))=0$. Hence,

$$
\begin{aligned}
\left|\tilde{E}_{h}(u)-E_{h}(u)\right| & \leq C h_{0}^{n+1} \sum_{i} \int_{e_{i}}\left|D^{n+1}(g(u))\right| d \mathbf{r}+\left|\tilde{J}_{h}(u)-J_{h}(u)\right| \\
& \leq C h_{0}\left\|g^{\prime}(u)\right\|_{L^{2}(\Omega)}|| \nabla u \|_{L^{2}(\Omega)}+\left|\tilde{J}_{h}(u)-J_{h}(u)\right| \text { (Inverse Inequality) }
\end{aligned}
$$

Lets denote by hypothesis $H 2$ the following conditions,

1. $f$ is a polynomial function of degree $d$ which satisfies the condition $n-d(k-1) \geq 0$

2. If $\left(u_{h}\right) \in\left(X_{h}\right)$ is a sequence such that $u_{h} \rightarrow u$ in $X$, then $\left\|g^{\prime}\left(u_{h}\right)\right\|_{L^{2}(\Omega)}$ is bounded uniformly

3. $N<4$

4. $n-2 k+3>0$

5. $p \geq 2$

Lemma 17 If $\left(u_{h}\right) \in\left(X_{h}\right)$ is a sequence such that $u_{h} \rightarrow u$ in $X$, and hypothesis $H$ and $H 2$ are satisfied, then $\lim _{h \rightarrow \infty}\left\{\tilde{E}_{h}\left(u_{h}\right)-E_{h}\left(u_{h}\right)\right\}=0$.

Proof. Follows from (25), Theorem 9 and Theorem 16. 
Theorem 18 If the hypothesis $H$ and $H 2$ are satisfied, then $\tilde{E}_{h} \rightarrow^{\Gamma} E$ in the weak topology of $X$.

Proof. let $\left(u_{h}\right)$ be a sequence such that $u_{h} \rightarrow u$ in $X$. We then have 2 cases.

Case1: There is no sub-sequence $\left(u_{h_{k}}\right)$ such that $\left(u_{h_{k}}\right) \in X_{h_{k}}$ $\liminf _{h \rightarrow \infty} \tilde{E}_{h}\left(u_{h}\right)=+\infty$. Hence, $\liminf _{h \rightarrow \infty} \tilde{E}_{h}\left(u_{h}\right) \geq E(u)$.

Case $2: \exists$ sub-sequence $\left(u_{h_{k}}\right)$ such that $\left(u_{h_{k}}\right) \in X_{h_{k}}$ $\liminf _{h \rightarrow \infty} \tilde{E}_{h}\left(u_{h}\right) \geq \liminf \inf _{h_{k} \rightarrow \infty} E_{h_{k}}\left(u_{h_{k}}\right)+\liminf _{h \rightarrow \infty}\left(\tilde{E}_{h_{k}}-E_{h_{k}}\right)\left(u_{h_{k}}\right)$ and by using Lemma 17 we get, $\liminf _{h \rightarrow \infty}\left(\tilde{E}_{h_{k}}-E_{h_{k}}\right)\left(u_{h_{k}}\right)=0$.

Hence, $\liminf \operatorname{in}_{h \rightarrow \infty} \tilde{E}_{h}\left(u_{h}\right) \geq \liminf _{h_{k} \rightarrow \infty} E_{h_{k}}\left(u_{h_{k}}\right) \geq E(u)$ (from Theorem 11).

Now we construct the recovery sequence from interpolated functions. Let $\left(u_{h}\right)$ be a sequence constructed from the interpolation functions of successive triangulations such that, $u_{h} \rightarrow u$ in $X . \lim _{h \rightarrow \infty} \tilde{E}_{h}\left(u_{h}\right)=\lim _{h \rightarrow \infty} E_{h}\left(u_{h}\right)+\lim _{h \rightarrow \infty}\left(\tilde{E}_{h}-E_{h}\right)\left(u_{h}\right)$. But $\lim _{h \rightarrow \infty}\left(\tilde{E}_{h}-E_{h}\right)\left(u_{h}\right)=0$ from Lemma 17. Hence, $\lim _{h \rightarrow \infty} \tilde{E}_{h}\left(u_{h}\right)=\lim _{h \rightarrow \infty} E_{h}\left(u_{h}\right)=$ $E(u)$. Hence, $\tilde{E}_{h} \rightarrow^{\Gamma} E$ in weak topology of $X$.

Lemma 19 If $f$ is a polynomial function of degree $d$ which satisfies the condition $n-$ $d(k-1) \geq 0, p \geq 2$ and $N\left(\max \left\{0, \frac{p-1}{p}-\frac{1}{2}\right\}\right)<1$ then, $\tilde{E}_{h}$ is equi-coercive in the weak topology of $X$.

Proof. First we note the following property about quadratures. If $A(u)=\int f(u), B(u)=$ $\int g(u)$ and $f(u(\mathbf{r})) \geq g(u(\mathbf{r}))$ on $\Omega$, then $\tilde{A}(u) \geq \tilde{B}(u)$. Hence, if $u \in X_{h}$, as $J_{h}(u) \geq 0$ and $q \geq p$ we have,

$$
\begin{array}{r}
E_{h}(u) \geq \int_{\Omega}\left\{f(\nabla u)+C_{1}|u|^{p}-C_{2}\right\} d \mathbf{r} \\
\tilde{E}_{h}(u) \geq Q\left\{\int_{\Omega}\left\{f(\nabla u)+C_{1}|u|^{p}-C_{2}\right\} d \mathbf{r}\right\}
\end{array}
$$


where, Q denotes the quadrature of the term inside the bracket. Hence,

$$
\begin{aligned}
\tilde{E}_{h}(u) & \geq \int_{\Omega}\left\{f(\nabla u)+C_{1}|u|^{p}\right\} d \mathbf{r}-C h_{0}\|u\|_{L^{(2 p-2)}(\Omega)}^{p-1}\|\nabla u\|_{L^{2}(\Omega)}-C_{2} \\
& \geq c_{0}\|\nabla u\|_{L^{p}(\Omega)}^{p}+C_{1}\|u\|_{L^{p}(\Omega)}^{p}-C h_{0}\|u\|_{L^{(2 p-2)}(\Omega)}^{p-1}\|\nabla u\|_{L^{2}(\Omega)}-C_{2} \\
& \geq c_{0}\|\nabla u\|_{L^{p}(\Omega)}^{p}+C_{1}\|u\|_{L^{p}(\Omega)}^{p}-C h_{0}^{1-N\left(\max \left\{0, \frac{p-1}{p}-\frac{1}{2}\right\}\right)}\|u\|_{L^{p}(\Omega)}^{(p-1)}\|\nabla u\|_{L^{2}(\Omega)}-C_{2}
\end{aligned}
$$

As $N\left(\max \left\{0, \frac{p-1}{p}-\frac{1}{2}\right\}\right)<1, \exists$ a $m$ such that $\forall h>m$,

$$
\tilde{E}_{h}(u) \geq K_{0}\|\nabla u\|_{L^{p}(\Omega)}^{p}+K_{1}\|u\|_{L^{p}(\Omega)}^{p}-K_{2}
$$

where, $K_{0}>0, K_{1}>0, K_{2}$ are constants independent of $h$. If $u \notin X_{h}$, then $\tilde{E}_{h}(u)=$ $+\infty$. Thus, the above expression is true for any $u$. It is now straightforward to show that $\left(\tilde{E}_{h}\right)$ is equi-coercive in the weak topology of $W^{1, p}(\Omega)$ and from Lemma 1, equi-coercive in the weak topology of $X$.

Theorem 20 If the hypothesis $H$ and $H 2$ are satisfied, and $N\left(\max \left\{0, \frac{p-1}{p}-\frac{1}{2}\right\}\right)<1$, then $\lim _{h \rightarrow \infty} \inf _{X} \tilde{E}_{h}=\min _{X} E$.

Proof. Follows from Lemma 19 and Theorem 18 and Theorem 7.8, Dal Maso (1993).

For the orbital-free energy functional, it is easy to check that it satisfies the following conditions:

1. $f$ is a polynomial function of degree 2 .

2. If $\left(u_{h}\right) \in\left(X_{h}\right), u_{h} \rightarrow u$ in $X$, then $\left\|g^{\prime}\left(u_{h}\right)\right\|_{L^{2}(\Omega)}$ is uniformly bounded, which follows from the continuity of $g^{\prime}$ and compact injection of $X$ in $L^{2 q-2}(\Omega)$.

3. $N\left(\max \left\{0, \frac{p-1}{p}-\frac{1}{2}\right\}\right)<1($ as $N=3, p=2)$.

Hence, if we choose an appropriate quadrature rule, all the results in this section will carryover to the orbital-free energy functional under consideration. 


\section{Numerical Implementation}

We now turn to a numerical implementation of the variational formulation (21) described in Section 2. We discretize the variational problem using a finite-element method and use a nested sequence of iterative conjugate-gradient solvers to solve for the electrostatic potential, charge density and atomic positions. For a given set of atomic positions, we relax the electron density, and for each electron density, we relax the electrostatic potential. An effective implementation of this procedure requires care with two aspects.

First, the electrostatic potential has to be solved on all space $\mathbb{R}^{3}$ while the electron density is solved only on a compact region $\Omega$. Since all the charges are confined to $\Omega$, the electrostatic potential will decay better than $1 / r$ since we have charge neutrality. We take advantage of this, and compute the electrostatic potential on a larger domain $\Omega^{\prime}$ satisfying, $\Omega^{\prime} \supset \Omega$, and impose zero Dirichlet boundary conditions on the boundary of the larger domain. Typically we use $\operatorname{dia}\left(\Omega^{\prime}\right) \approx 10^{2} \operatorname{dia}(\Omega)$ in our calculations. Further, we coarsen our mesh as we go away from $\Omega$ to keep the computations efficient and accurate.

Second, we anticipate that the charge density and the electrostatic potential will be localized near the atomic cores, and to be convected along with the cores as the atomic positions change. In other words, we anticipate that the spatial perturbation of the electron density would be large as the atomic positions change, but the perturbation to be small in a coordinate system that is convected with the atomic position. Therefore, with each update of the atomic position we convect the finite-element grid and as well as the old electron density and electrostatic potential, and use this convected electron density and potential as an initial guess for the subsequent iteration.

We implement these two aspects in the following way by using two triangulations. We first construct a coarse or atomistic triangulation $\mathrm{T}$ of the large domain $\Omega^{\prime}$ with $K$ nodal 
points located at $\left\{\mathbf{x}_{i}\right\}_{i=1}^{K}$. This triangulation contains each initial atomic position as a node so that it has atomic resolution in the small region $\Omega$, and coarsens away from it. We use a coarsening rate of $r^{6 / 5}$ which is estimated to be optimal for a $1 / r$ decay with linear interpolation. The triangulation is generated automatically from Delaunay triangulation of a set of points. This is shown in Figure 1. We now introduce a second triangulation $\mathrm{T}^{\prime}$ which is a uniform subdivision of $\mathrm{T}$ repeated a certain number of times by using the Freudenthal's algorithm for a 3-simplex (Bey, 2000). This triangulation is sufficiently fine to resolve the electronic charges and the electrostatic field, and is shown in Figures 2 and 3. At any step in the iteration suppose $\varphi^{i}: \mathbb{R}^{3} \rightarrow \mathbb{R}^{3}$ denote the deformation of the $i$ th atom. We extend this deformation mapping to all nodes of the triangulation $\mathrm{T}$ by setting it to zero for nodes that do not coincide with atomic positions, and then use a linear interpolation to extend this deformation to $\Omega^{\prime}$ :

$$
\varphi(\mathbf{x})=\sum_{i=1}^{n} \varphi^{i} N_{i}(\mathbf{x})
$$

where $N_{i}$ is the shape-function associated with the $i$ th node and $n$ is the number of vertices in the simplex associated with triangulation $\mathrm{T}$. We use this deformation to deform the fine mesh $\mathrm{T}^{\prime}$. Specifically, we define a new mesh $\mathrm{T}^{\prime \varphi}$ with nodes

$$
\mathbf{x}_{a}^{\varphi}=\varphi\left(\mathbf{x}_{a}\right)=\sum_{i=1}^{n} \varphi^{i} N_{i}\left(\mathbf{x}_{a}\right) \quad a=1, \ldots, L
$$

where $\mathbf{x}_{a}$ are the position of the nodes of the original triangulation $\mathrm{T}^{\prime}$ and $L$ are the number of such nodes.

We use this mesh, $\mathrm{T}^{\prime \varphi}$ to discretize the electron density and electrostatic potential. It consists of 4-node tetrahedral elements and the interpolating shape functions are linear. We use a 4-point Gaussian quadrature which is second order accurate and satisfies (1) \& (4) in hypothesis $H 2$. So the results of Section 5 hold. We solve the finite-element equations using non-linear conjugate gradients with secant method for line search. However, since 
the mesh is adapted to the updated atomic positions, and the electron density convected from the previous atomic position (by keeping the nodal values constant while the mesh deforms) is used as an initial guess, the convergence is rapid. Finally, we implement the computation in parallel using domain decomposition.

It is possible that the quality of the triangulation could deteriorate and the aspect ratio of the elements become very small as the mesh deforms. To work around this, with each update of $\mathrm{T}^{\prime \varphi}$, we evaluate the minimum value of the aspect ratio (defined as ratio of the radii of inscribed sphere to the circumsphere) amongst all elements, and remesh the region with the nodes fixed if it is below a prescribed value.

\section{Examples}

The approach presented is demonstrated and tested by means of simulations performed on atoms, molecules and clusters of aluminum.

\subsection{Atoms}

The first test case is the hydrogen atom for which theoretical results are available. We use a value of $\lambda=\frac{1}{3}$ since it gives the best results. Figure 4 demonstrates the convergence of our finite-element approach. We use $N_{0} \approx 100$ elements for the initial mesh and have $N_{0} 8^{n}$ elements after the $n^{\text {th }}$ subdivision. It shows that the ground state energy converges almost exponentially as the number of subdivisions (i.e., the fine-ness of the triangulation) is increased. It also shows that the ground state energy of the hydrogen atom is computed to be -0.495 Hartree as against the theoretical value of -0.5 Hartree. Figure 5 shows the radial distribution of the electron density around the hydrogen nucleus. It is compared with the theoretical solution obtained by solving the Schrödinger equation. The comparison is 
very good except at the regions very close to the nucleus, where the simulations predict a slightly higher electron density. Figure 6 shows the radial probability distribution of finding the electron as a function of the distance from the nucleus. We observe that the probability of finding the electron is maximum at a distance of 1 Bohr from the nucleus which agrees with the theoretical solution.

To simulate atoms heavier than hydrogen atom, $\lambda=\frac{1}{9}$ is used, which is the conventional gradient correction to Thomas-Fermi kinetic energy functional. The ground state energies of various other atoms estimated from our simulations, are tabulated in table 1 under DFTFE, which denotes orbital-free density functional calculation in a finite-element basis. The results obtained are compared with other ab inito calculations (Tong \& Sham, 1966; Clementi et al., 1962) which include, the Hartree-Fock approach and the Kohn-Sham approach of density functional theory using local density approximation for exchange correlation functionals (KS-LDA). The ground state energies are found to be in good agreement with other ab-initio calculations and experiments.

\subsection{Molecules}

The next set of examples we consider are $\mathrm{N}_{2}$ and $\mathrm{CO}$ molecules. The ground state energies of these molecules are evaluated at various values of interatomic distances. Using this data, the binding energies and bond lengths of the molecules are determined. Figure 7 shows the binding energy for $\mathrm{N}_{2}$ molecule as a function of the interatomic distance. The interatomic potential energy has the same form as other popular interatomic potentials like Leonard-Jones and Morse potentials. Tables $2 \& 3$ show the comparison of binding energies and bond lengths of $\mathrm{N}_{2}$ and $\mathrm{CO}$ molecules predicted from our simulations with those from other $a b$ inito calculations and experiments (Gunnarsson et al., 1977; Cade et al., 1973; Hou, 1965; Huber, 1972). There is reasonable agreement of our simulations 
with experiments in terms of the binding energies. But there is a considerable deviation in the values of predicted bond lengths in comparison to other calculations and experiments. We believe that this is due to the well-understood limitation of the orbital-free kinetic energy functionals in the presence of strong covalent bonds (Parr \& Yang, 1989).

\subsection{Aluminum Clusters}

The final set of examples we consider are aluminum clusters. We choose $\lambda=\frac{1}{6}$, which was found to yield good results. The simulations are performed using a modified form of Heine-Abarenkov pseudopotential for aluminum (Goodwin et al., 1990), which in real space has the form,

$$
V_{e x t}= \begin{cases}-\frac{Z_{v}}{r}, & \text { if } r \geq r_{c} \\ -A, & \text { if } r<r_{c}\end{cases}
$$

where, $Z_{v}$ is the number of valence electrons, $r_{c}$ the cut-off radius and $\mathrm{A}$ is a constant. For aluminum, $Z_{v}=3, r_{c}=1.16$ a.u., $A=0.11$ a.u.. Simulations are performed on clusters consisting of $1 \times 1 \times 1,3 \times 3 \times 3,5 \times 5 \times 5$ and $9 \times 9 \times 9$ face-centered-cubic (fcc) unit cells. The number of atoms in the cluster consisting of $9 \times 9 \times 9 \mathrm{fcc}$ unit cells is 3730 and close to 6 million finite-elements are used in this simulation. It took more than 10,000 CPU hours on $2.4 \mathrm{GHz}$ processors for each simulation on the cluster with $9 \times 9 \times 9 \mathrm{fcc}$ unit cells to convergence. Figures $8 \& 9$ show the contours of electron density for a cluster consisting of $3 \times 3 \times 3$ fcc unit cells. Figure 10 show the binding energy per atom as a function of the lattice constant (size of the fcc cell) for the various cluster sizes, along with cubic polynomial fits of the simulated points. We calculate the binding energy using the standard approach; $E_{\text {bind }}($ per atom $)=\left(E(n)-n E_{0}\right) / n$ where $E(n)$ is the energy of the cluster/unit cell containing $n$ atoms and $E_{0}$ is the energy of a single atom. An important 
observation from these figures is the anharmonic nature of the binding energy.

The binding energies, evaluated in these simulations include along with the bulk cohesive energy, the effects of surfaces, edges and corners. A classical interpretation of these energies would suggest a scaling of the form

$$
\epsilon_{n}=\epsilon_{\text {coh }}+n^{-1 / 3} \epsilon_{\text {surf }}+n^{-2 / 3} \epsilon_{\text {edge }}+n^{-1} \epsilon_{\text {corn }}
$$

where, $n$ represents the number of atoms, $\epsilon_{c o h}$ the cohesive energy of the bulk, $\epsilon_{\text {surf }}$ the surface energy, $\epsilon_{\text {edge }}$ the energy contributed by presence of edges and $\epsilon_{\text {corn }}$ the energy resulting from the corners. Figure 11 shows the plot of binding energy per atom of each cluster in the relaxed configuration as a function of $n^{-1 / 3}$. The relationship is almost linear, which supports the scaling relation given in (29). Further, it shows that cohesive and surface energies dominate edge and corners even for relatively small clusters. Finally, this scaling allows us to extract the bulk cohesive energy of aluminum from the binding energies of the clusters.

The values of the bulk modulus of these clusters are evaluated from the binding energy calculations. Figure 12 shows the linear dependence of bulk modulus on $n^{-1 / 3}$, implying that bulk modulus can also be expressed as a scaling relation suggested by (29).

Table 4 shows the variation of the lattice constant with the cluster size. We do not find significant dependence or a clear trend in the dependence of lattice constant on cluster size. Table 5 shows a comparison of the bulk properties of aluminum obtained from our simulations with other ab initio calculations (Goodwin et al., 1990) and experiments (Brewer, 1977; Gschneider, 1964). We have very good quantitative agreement in terms of both cohesive energies and bulk modulus. The lattice constant of $9 \times 9 \times 9$ cluster is 7.42 , which is very close to that predicted by KS-LDA which is 7.44 .

In all the simulations discussed so far, the ground state energy calculations were per- 
formed for fixed atomic positions. However, the formulation developed is capable of equilibrating the nuclear positions and predicting the various stable configurations of atoms. To this end, we perform simulations on small aluminum clusters to predict the binding energies and the equilibrated structures of these clusters. Since the energy is non-convex with respect to the positions of the nuclei, we start our simulations from various initial configurations to predict the stable configurations of these clusters. We performed simulations on small aluminum clusters consisting of two, three and four atoms. Table 6 shows the results of our simulations and comparison with other DFT calculations (Ahlrichs \& Elliot, 1999). We successfully predict the various stable configurations of these clusters and the binding energies of these clusters are in good agreement with other calculations. However, there is some deviation in the predicted geometry. This deviation could be attributed to the fact that the bonding in these small aluminum clusters is covalent in nature and orbital-free kinetic functionals are not very appropriate for systems with covalent bonds.

\section{Conclusion}

We have developed a non-periodic finite-element formulation of density functional calculations based on orbital-free kinetic energy functionals to perform ground state energy calculations. This formulation aids in addressing problems which are non-periodic in nature like defects in solids which can not be treated with justice with existing techniques employing periodic boundary conditions. The use of a finite-element basis and orbitalfree kinetic energy functionals enables us to solve large systems with thousands of atoms effectively, which has been demonstrated through simulations on large aluminum clusters. We have also established the convergence of the finite-element approximation using $\Gamma$-convergence. 
The method was tested by carrying out simulations on atoms, molecules and large clusters of aluminum in fcc structure. We have also predicted some stable structures in small aluminum clusters. The results from these simulations which include energies of atoms, binding energies and bond lengths of molecules, bulk properties of aluminum and stable configurations of small aluminum clusters along with their binding energies are compared with other $a b$ initio calculations and experiments. In most cases the agreement has been very good, except for molecules, where there is considerable deviation in the bond length predicted. This can be attributed to the inability of the orbital-free kinetic energy functionals to approximate the non-interacting kinetic energy well in systems with strong covalent bonding.

The present formulation is a step towards a larger goal of studying defects with long range interactions as are common in solids. This will require us to find a way to combine these non-periodic cluster calculations with classical continuum theories in a seamless manner. Similarly it will also require us to extend our approach to the exact Kohn-Sham density functional theory. These are the subject of current research.

\section{Acknowledgements}

The financial support of the Army Research Office under MURI Grant No. DAAD19-011-0517 is gratefully acknowledged. MO also gratefully acknowledges the support of the

Department of Energy through Caltech's ASCI ASAP Center for the Simulation of the Dynamic Response of Materials. 


\section{Appendix}

In this appendix we discuss briefly how the suggested approach can be extended to the family of kinetic energy functionals with kernel energy. The kernel energy is of the form,

$$
T_{k}(u)=\iint f(u(\mathbf{r})) K\left(\left|\mathbf{r}-\mathbf{r}^{\prime}\right|\right) g\left(u\left(\mathbf{r}^{\prime}\right)\right) d \mathbf{r} d \mathbf{r}^{\prime}
$$

Different types of kernel energies differ in the functional form of $f$ and $g$. However, most of them have same functional forms for $f$ and $g$. To keep the analysis simple we consider the case when $f$ and $g$ have the same functional form. Thus, the kernel energy can be written as,

$$
T_{k}(u)=\iint f(u(\mathbf{r})) K\left(\left|\mathbf{r}-\mathbf{r}^{\prime}\right|\right) f\left(u\left(\mathbf{r}^{\prime}\right)\right) d \mathbf{r} d \mathbf{r}^{\prime}
$$

Choly \& Kaxiras (2002) propose a real space approach to evaluate these integral by approximating the kernel in the reciprocal space by a rational function. Under this approximation, the kernel energy has a local form, given by,

$$
\begin{array}{r}
T_{k}(u)=\sum_{j=1}^{m} \frac{1}{2 C_{j}} Z_{j}(u)+\left(\sum_{j=1}^{m} P_{j}\right) \int_{\Omega} f(u)^{2} d \mathbf{r} \\
Z_{j}(u)=\inf _{w_{j} \in H_{0}^{1}(\Omega)} \frac{C}{2} \int_{\Omega}\left|\nabla w_{j}\right|^{2} d \Omega+\frac{Q_{j}}{2} \int_{\Omega} w_{j}^{2} d \Omega+C_{j} \int_{\Omega} w_{j} f(u) d \Omega \quad j=1, \ldots m
\end{array}
$$

where, $C$ is a positive constant, $C_{j}, Q_{j}$ are constants determined from the fitted rational function with degree $2 m$. The minimization in (.1) is well defined if $\frac{C}{C_{\Omega}}+Q_{j}>0$, where $C_{\Omega}$ is the constant from Poincaré inequality. This can be easily verified using Poincaré inequality and Lax-Milgram Lemma.

The common functional form of $f$ used in the kernel energy is $f=u^{2 \alpha}$. For this functional form its easy to verify, following the same recipe used to treat the electrostatic interaction 
energy from sections $3,4 \& 5$, that all the previous mentioned results hold if $\alpha<2$. Other functional forms of $f$ must be treated on a more specific level. 


\section{References}

Hohenberg, P., Kohn, W., 1964. Inhomogeneous electron gas. Phys. Rev. 136, B864.

Kohn, W., Sham, L.J., 1965. Self-consistent equations including exchange and correlation effects. Phys. Rev. 140, A1133.

Finnis, M., 2003. Interatomic forces in condensed matter, Oxford University Press, New York.

Parr, R.G., Yang, W., 1989. Density-functional theory of atoms and molecules, Oxford University Press, New York.

Pask, J.E., Klein, B.M., Fong, C.Y., Sterne, P.A., 1999. Real-space local polynomial basis for solid-state electronic structure calculations: A finite-element approach. Phys. Rev. B 59, 12352.

Hehre, W.J., Stewart, R.F., Pople J.A., 1969. Self-consistent molecular-orbital methods .I. use of gaussian expansions of slater-type atomic orbitals. J. Chem. Phys. 51, 2657.

Wills, J.M., Copper, B.R., 1987. Synthesis of band and model hamiltonian theory for hybridizing cerium systems. Phys. Rev. B 36, 3809.

Soler et al. 2002. The SIESTA method for ab initio order-N materials simulation. J. Phys. Condens. Mat. 14, 2745.

Skylaris, C.K., Haynes, P.D., Mostofi, A.A., Payne, M.C., 2005. Linear-scaling density functional simulations on parallel computers. J. Chem. Phys. 122, 084119.

Bowler, D.R., Choudhury, R., Gillan, M.J., Miyazaki, T., 2006. Recent progress with large-scale ab initio calculations: the CONQUEST code. Physica Status Solidi B 243, 989.

Thomas, L.H., 1927. The calculation of atomic fields. Proc. Cambridge Phil. Soc. 23, 542.

Fermi, E., 1927. Un metodo statistice per la determinazione di alcune proprieta dell'atomo. Rend. Acad. Lincei 6, 602.

Wang Y.A., Govind, N., Carter, E.A., 1998. Orbital-free kinetic-energy functionals for the 
nearly free electron gas. Phys. Rev. B 58, 13465.

Wang Y.A., Govind, N., Carter, E.A., 1999. Orbital-free kinetic-energy density functionals with a density-dependent kernel. Phys. Rev. B 60, 16350.

Smargiassi, E., Madden, P.A., 1994. Orbital-free kinetic-energy functionals for firstprinciple molecular dynamics. Phys. Rev. B 49, 5220.

Wang, L., Teter, M.P., 1992. Kinetic energy functional of electron density. Phys. Rev. B, $45,13196$.

De Giorgi, E., Franzoni, T., 1975. Su un tipo di convergenza variazionale. Atti Acad. Naz. Linccei Rend. Cl. Sci. Mat. 58, 842.

Gianni Dal Maso, 1993. An introduction to $\Gamma$-convergence, Birkhäuser, Boston.

Ceperley, D.M., Alder, B.J., 1980. Ground state of the electron gas by a stochastic method. Phys. Rev. 45, 566.

Perdew, J.P., Zunger, A., 1981. Self-interaction correction to density-functional approximation for many-electron systems. Phys. Rev. B 23, 5048.

Braides, A., 2002. $\Gamma$-convergence for beginners, Oxford University Press, New York.

Ciarlet, P.G., 2002. The finite element method for elliptic problems, SIAM, Philadelphia.

Bey, J., 2000. Simplicial grid refinement: on Freudenthal's algorithm and the optimal number of congruence classes. Numer. Math. 85, 1.

Tong, B.Y., Sham, L.J., 1966. Application to a self-consistent scheme including exchange and correlation effects to atoms. Phys. Rev. 144, 1.

Clementi, E., Roothaan, C.C.J., Yoshimine, M., 1962. Accurate analytical self-consistent field functions for atoms. II. Lowest configurations of neutral first row atoms. Phys. Rev. 127, 1618.

Gunnarsson, O., Harris, J., Jones, R.O., 1977. Density functional theory and molecular bonding. I. First-row diatomic molecules. J. Chem. Phys. 67, 3970.

Cade, P.E., Sales, K.D., Wahl, A.C., 1973. Electronic structure of diatomic molecules. 
III.A. Hartree-Fock wave functions and energy quantities for $\mathrm{N}_{2}$ and $\mathrm{N}_{2}^{+}$molecular ions. J. Chem. Phys. 44, 1973.

Hou, W.M., 1965. Electronic structure of CO and BF. J. Chem. Phys. 43, 624.

Huber, K.P., 1972. Constants of diatomic molecules, in American institute of physics handbook, McGraw-Hill, New York.

Goodwin, L., Needs, R.J., Heine, V., 1990. A pseudopotential total energy study of impurity promoted intergranular embrittlement. J. Phys. Condens. Matter 2, 351.

Brewer, L., 1977. Lawrence Berkeley labaratory report No. 3720 (unpublished)

Gschneider, K.A., 1964. Solid state physics, New York: Academic vol 16, 276.

Ahlrichs, R., Elliot, S.D., 1999. Clusters of aluminum, a density functional study. Phys. Chem. Chem. Phys. 1, 13.

Choly, N., Kaxiras, E., 2002, Kinetic energy density functionals for non-periodic systems. Solid State Comm. 121, 281. 
Table 1

Energies of atoms, computed by various techniques, in atomic units

\begin{tabular}{|c|c|c|c|c|}
\hline Element & DFT-FE & KS-LDA & Hartree-Fock & Experiments \\
\hline $\mathrm{He}$ & -2.91 & -2.83 & -2.86 & -2.9 \\
\hline $\mathrm{Li}$ & -7.36 & -7.33 & -7.43 & -7.48 \\
\hline $\mathrm{Ne}$ & -123.02 & -128.12 & -128.55 & -128.94 \\
\hline
\end{tabular}

Table 2

Binding energy and bond length of $\mathrm{N}_{2}$ molecule, computed by various techniques

\begin{tabular}{|c|c|c|c|c|}
\hline Property & DFT-FE & KS-LDA & Hartree-Fock & Experiments \\
& & (Gunnarsson et al., 1977) & (Cade et al., 1973) & (Huber, 1972) \\
\hline Binding energy (eV) & -11.9 & -7.8 & -5.3 & -9.8 \\
\hline Bond length (a.u.) & 2.7 & 2.16 & 2.01 & 2.07 \\
\hline
\end{tabular}

Table 3

Binding energy and bond length of $\mathrm{CO}$ molecule, computed by various techniques

\begin{tabular}{|c|c|c|c|c|}
\hline Property & DFT-FE & KS-LDA & Hartree-Fock & Experiments \\
& & (Gunnarsson et al., 1977) & (Hou, 1965) & (Huber, 1972) \\
\hline Binding energy (eV) & -12.6 & -9.6 & -7.9 & -11.2 \\
\hline Bond length (a.u.) & 2.75 & 2.22 & 2.08 & 2.13 \\
\hline
\end{tabular}


Table 4

Relaxed lattice constants of various cluster sizes, computed using DFT-FE

\begin{tabular}{|c|c|c|c|c|}
\hline Cluster size & $1 \times 1 \times 1$ & $3 \times 3 \times 3$ & $5 \times 5 \times 5$ & $9 \times 9 \times 9$ \\
\hline Relaxed lattice constant (a.u.) & 7.26 & 7.27 & 7.39 & 7.42 \\
\hline
\end{tabular}

Table 5

Bulk properties of aluminum, computed using various techniques

\begin{tabular}{|c|c|c|c|}
\hline Bulk Property & DFT-FE & KS-LDA & Experiments \\
& & (Goodwin et al., 1990) & (Brewer, 1977; Gschneider, 1964) \\
\hline Cohesive energy (eV) & 3.69 & 3.67 & 3.4 \\
\hline Bulk modulus (GPa) & 83.1 & 79.0 & 74.0 \\
\hline
\end{tabular}

Table 6

Comparison of properties of aluminum clusters $\mathrm{Al}_{n}, n=2,3,4$, obtained from DFT-FE calculations with other DFT calculations; $G$ denotes the symmetry group, $E_{b}$ denotes the binding energy per atom (eV), $R_{e}$ denotes equilibrium distances (a.u.)

\begin{tabular}{|l|l|ll|ll|}
\hline $\mathrm{n}$ & $\mathrm{G}$ & \multicolumn{2}{|l|}{ DFT-FE } & \multicolumn{2}{l|}{ AE (Ahlrichs \& Elliot, 1999) } \\
& & $E_{b}$ & $R_{e} /$ angle & $E_{b}$ & $R_{e} /$ angle \\
\hline 2 & $D_{\infty h}$ & -0.86 & 4.97 & -0.78 & 4.72 \\
\hline 3 & $D_{3 h}$ & -1.24 & 5.06 & -1.29 & 4.77 \\
\hline 3 & $C_{2 v}$ & -1.16 & 5.14 & -1.22 & 4.91 \\
\hline 4 & $D_{2 h}$ & -1.38 & $5.22 / 71^{\circ}$ & -1.5 & $4.85 / 68^{\circ}$ \\
\hline 4 & $C_{3 v}$ & -1.31 & & -1.39 \\
\hline
\end{tabular}




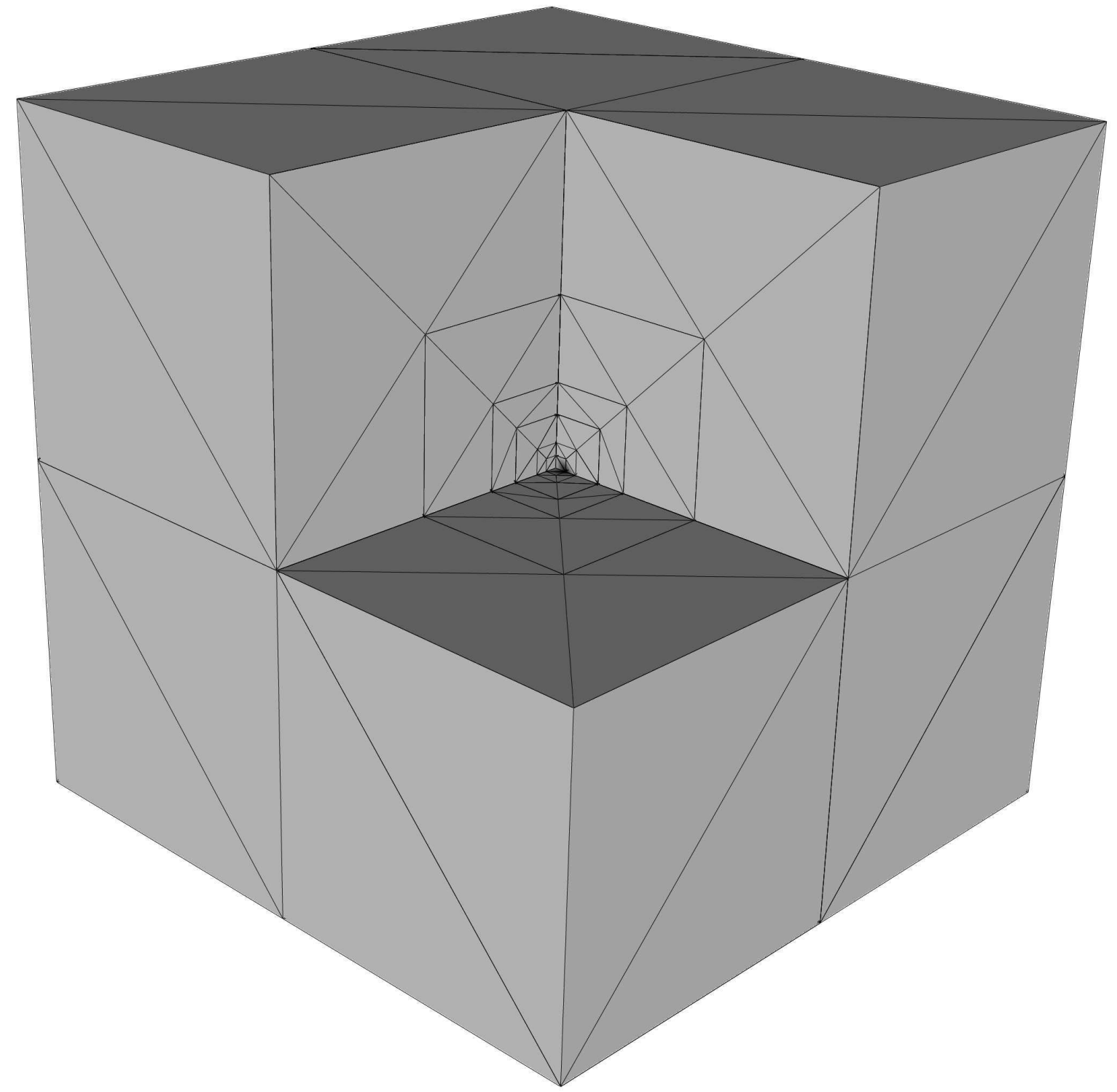

Fig. 1. Surface mesh of a sliced cubical domain corresponding to the triangulation $\mathrm{T}$ 


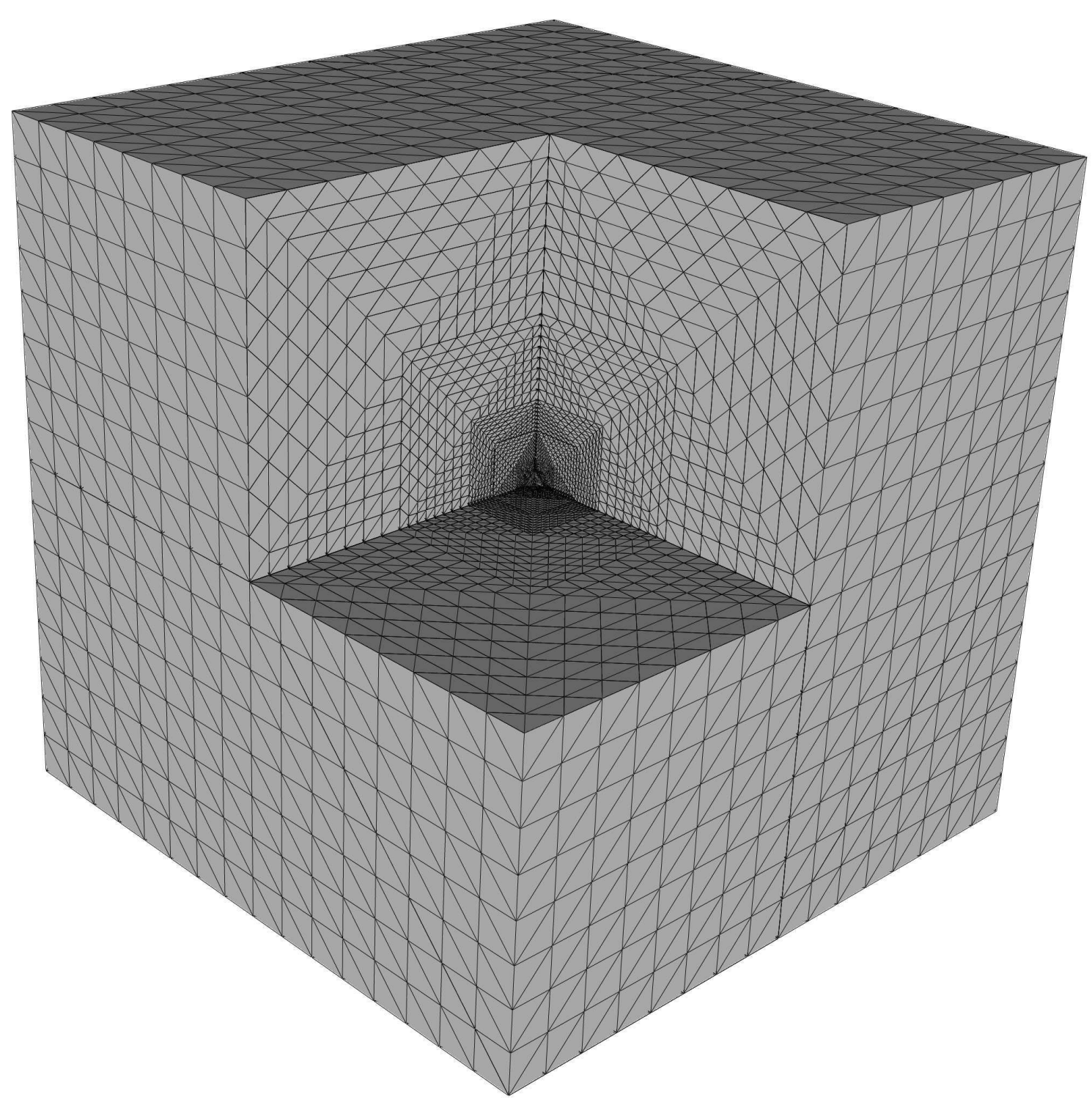

Fig. 2. Surface mesh of a sliced cubical domain corresponding to the triangulation $T^{\prime}$ 


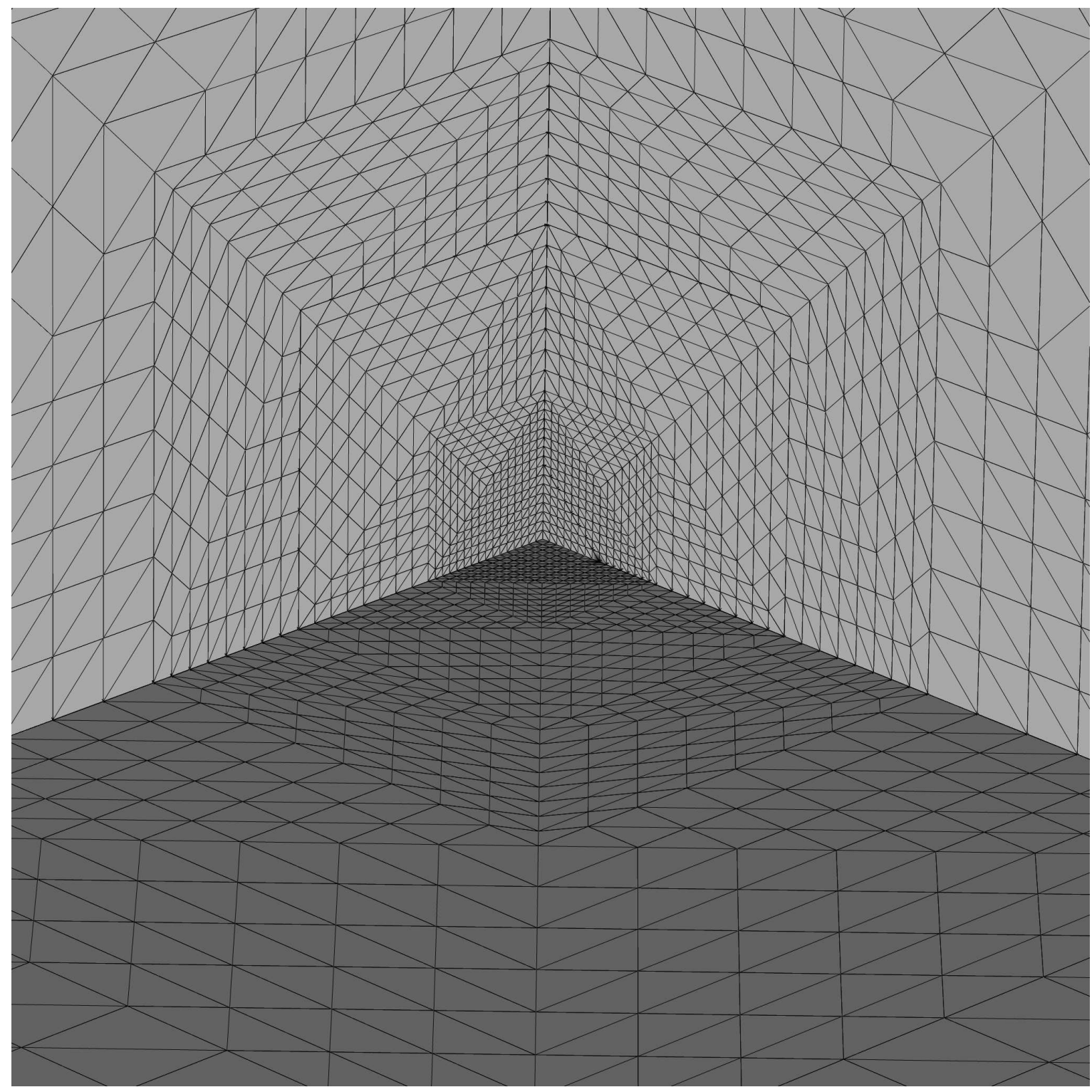

Fig. 3. Close up of figure 2 


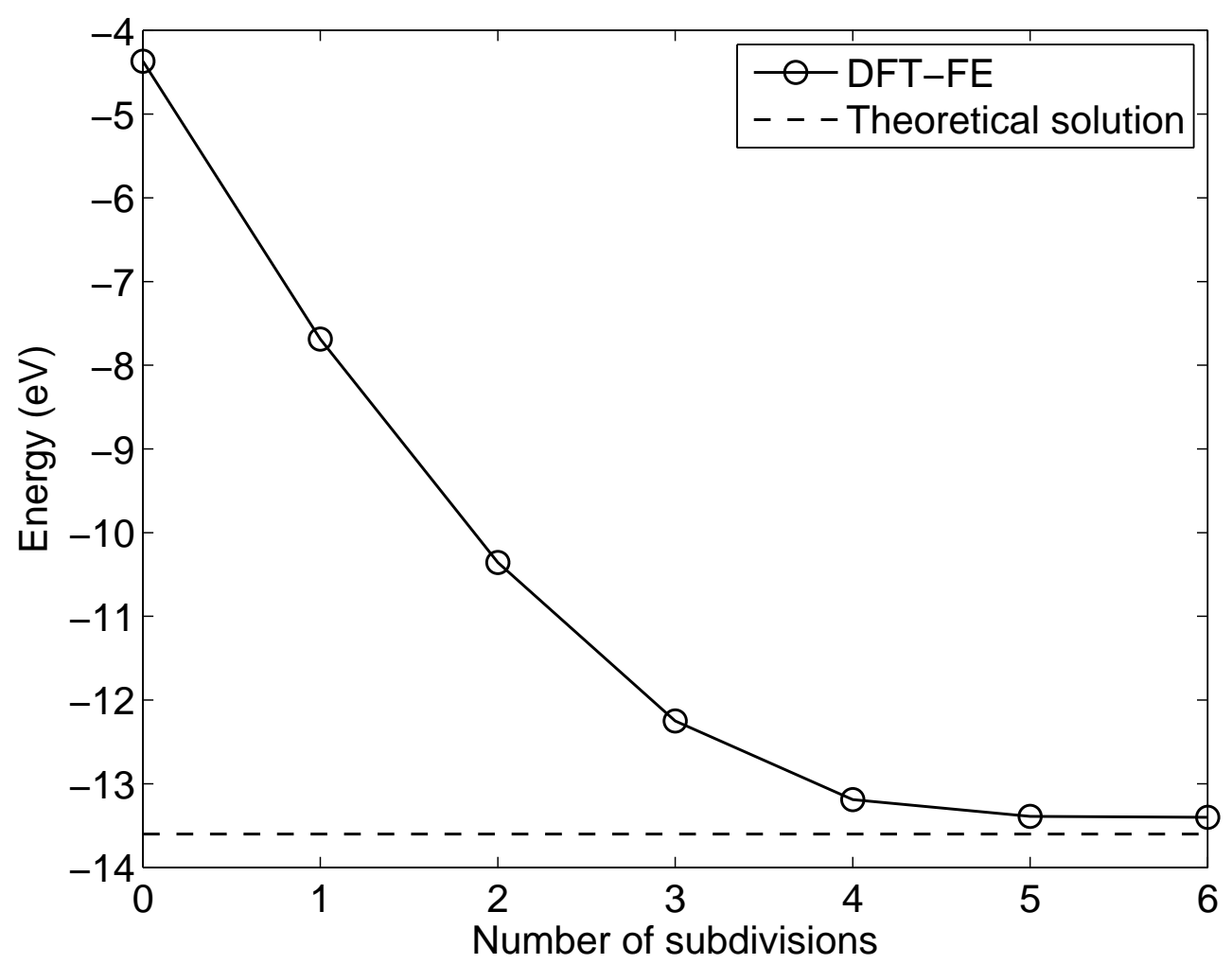

Fig. 4. Energy of hydrogen atom as a function of number of uniform subdivisions of triangulation $\mathrm{T}$ 


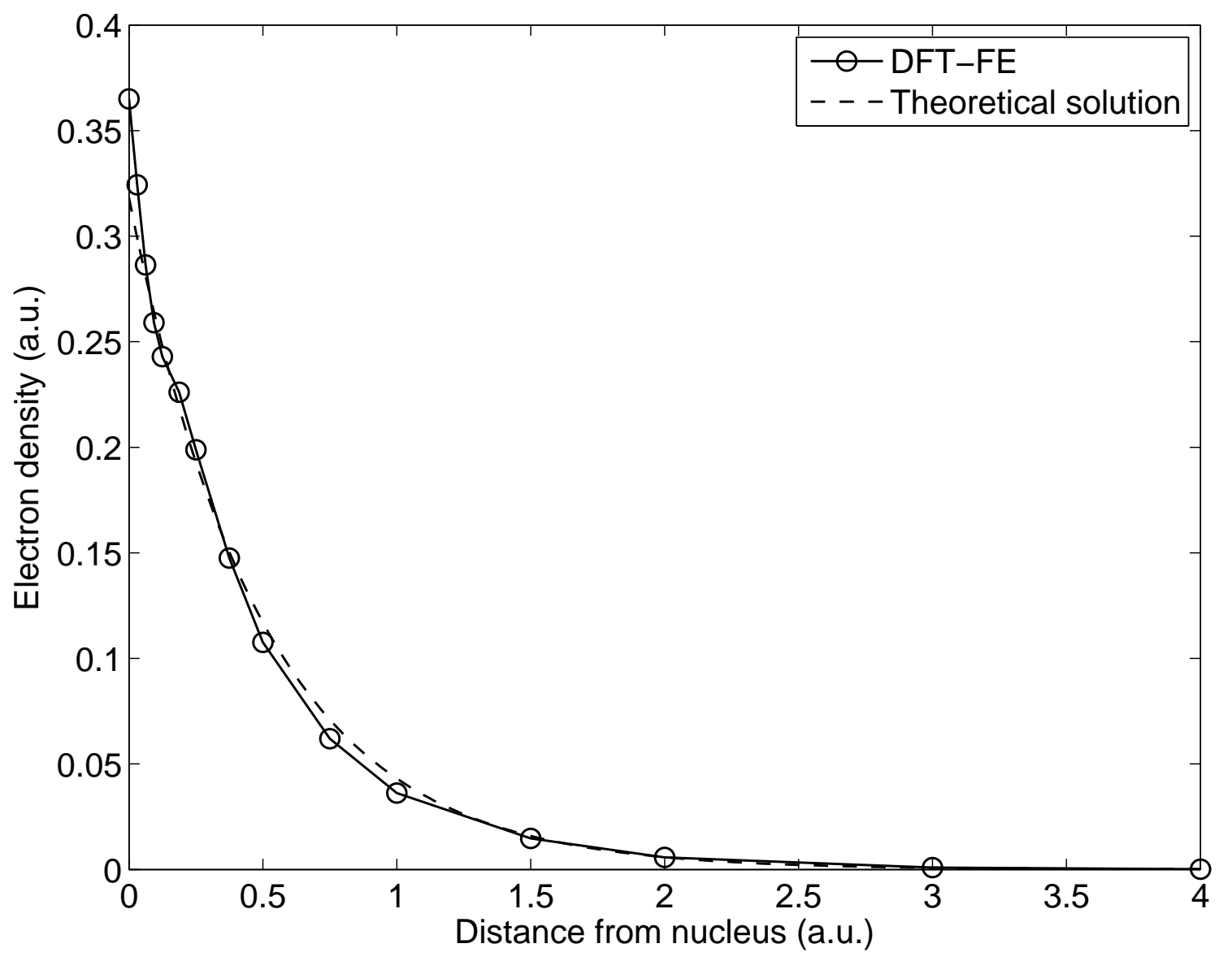

Fig. 5. Radial distribution of electron density for hydrogen atom 


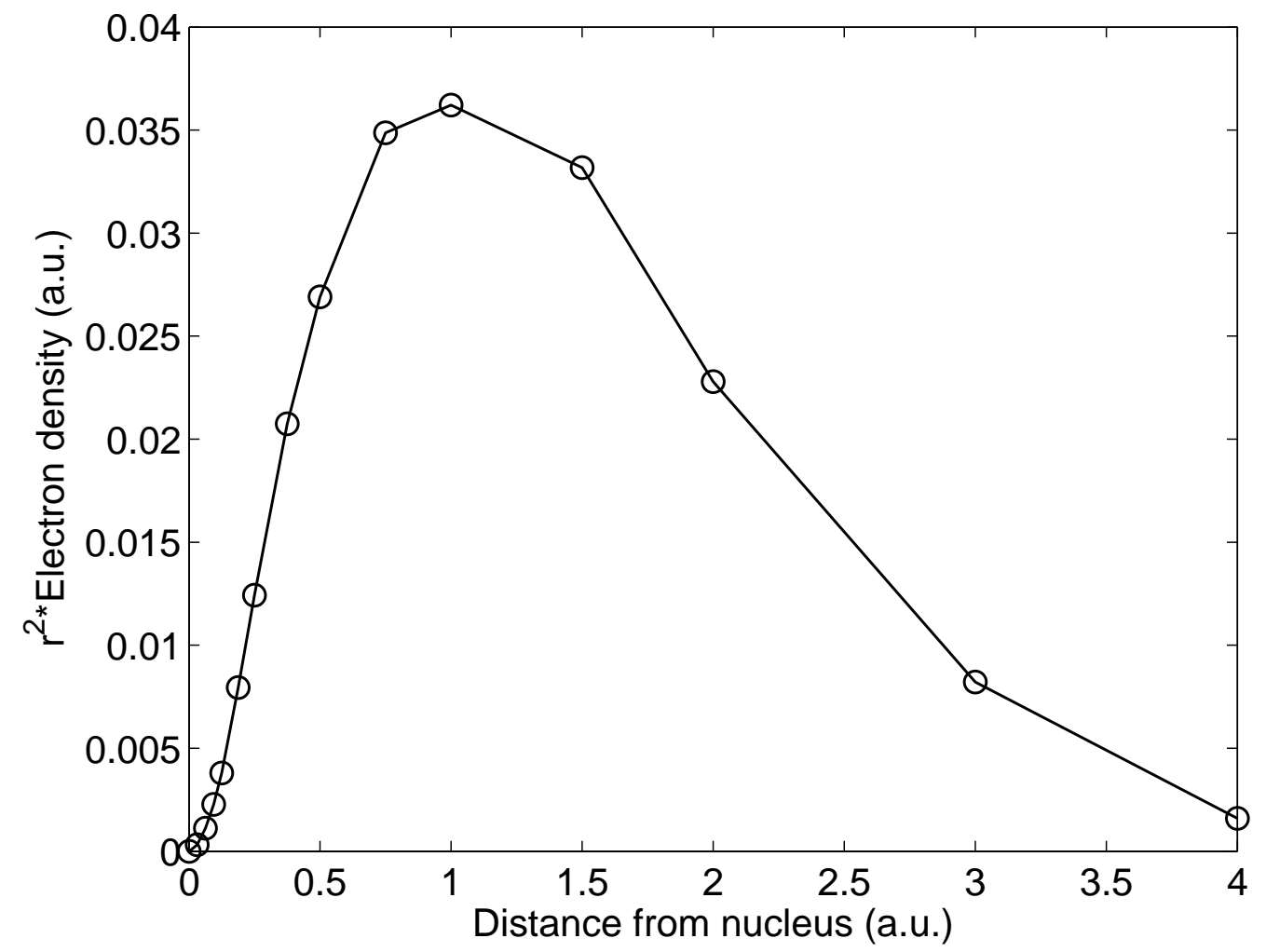

Fig. 6. Radial probability distribution of finding an electron around the hydrogen nucleus, computed using DFT-FE 


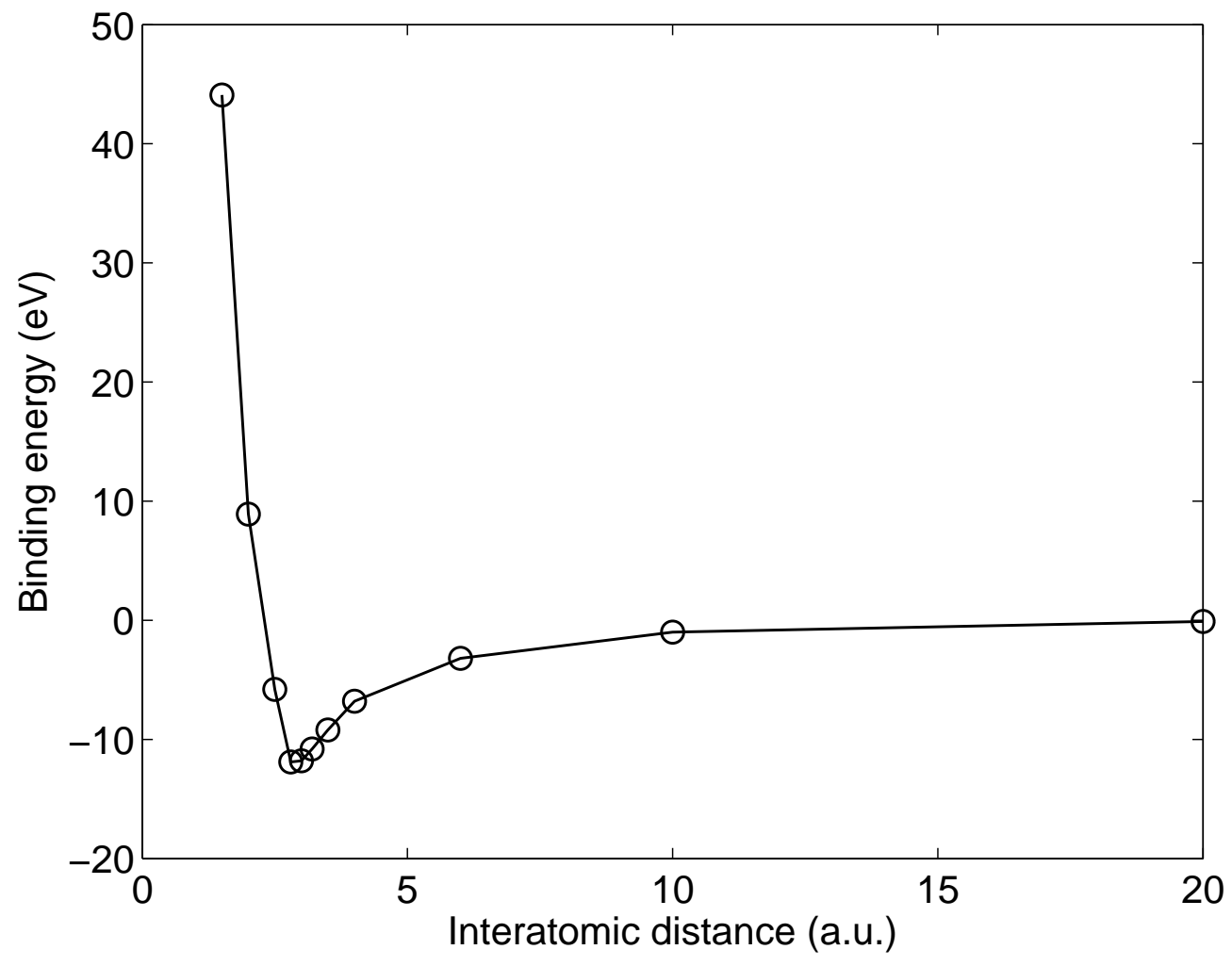

Fig. 7. Binding energy of $\mathrm{N}_{2}$ molecule as a function of interatomic distance, computed using DFT-FE 


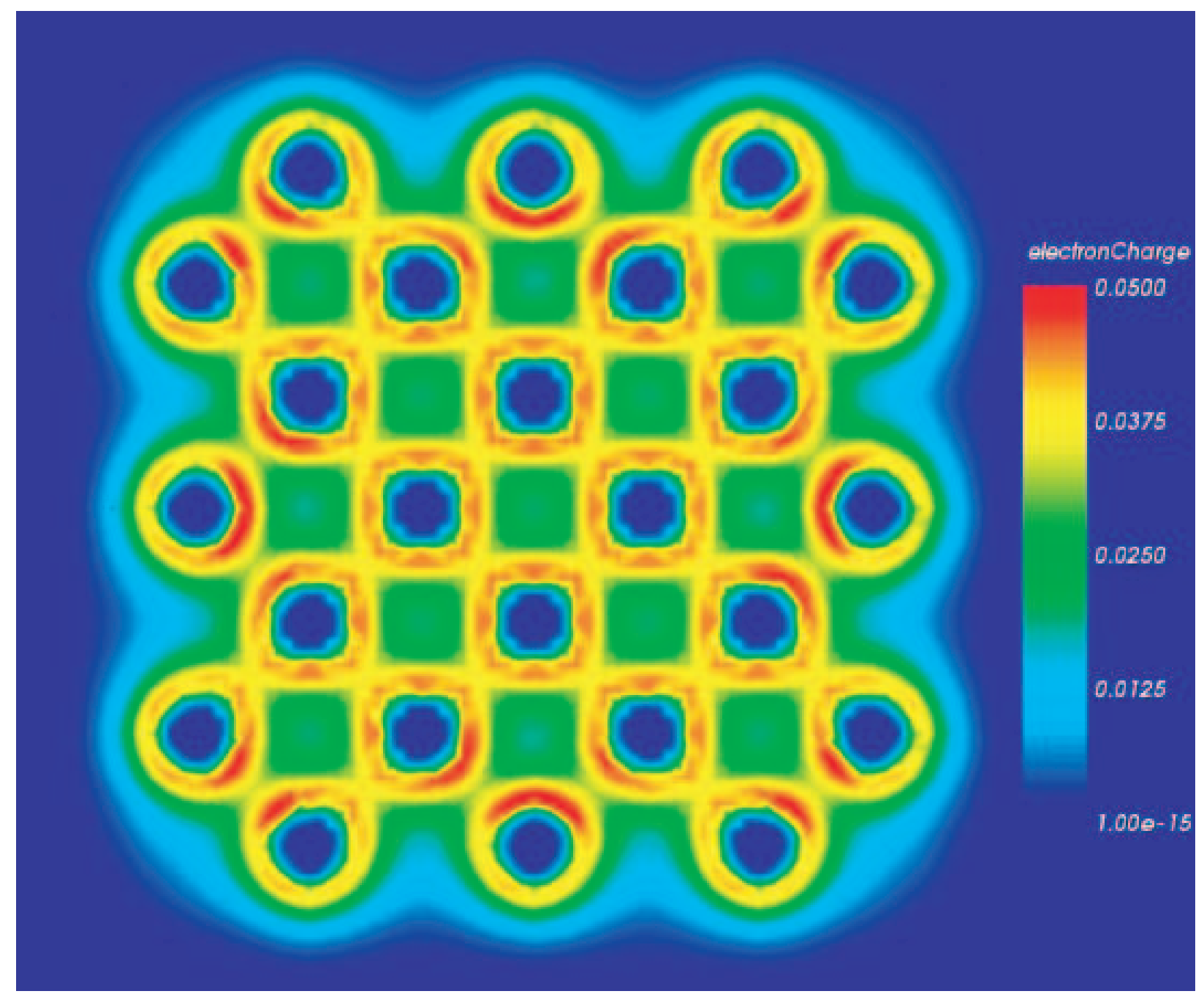

Fig. 8. Contours of electron density on the mid plane of an aluminum cluster with $3 \times 3 \times 3 \mathrm{fcc}$ unit cells 


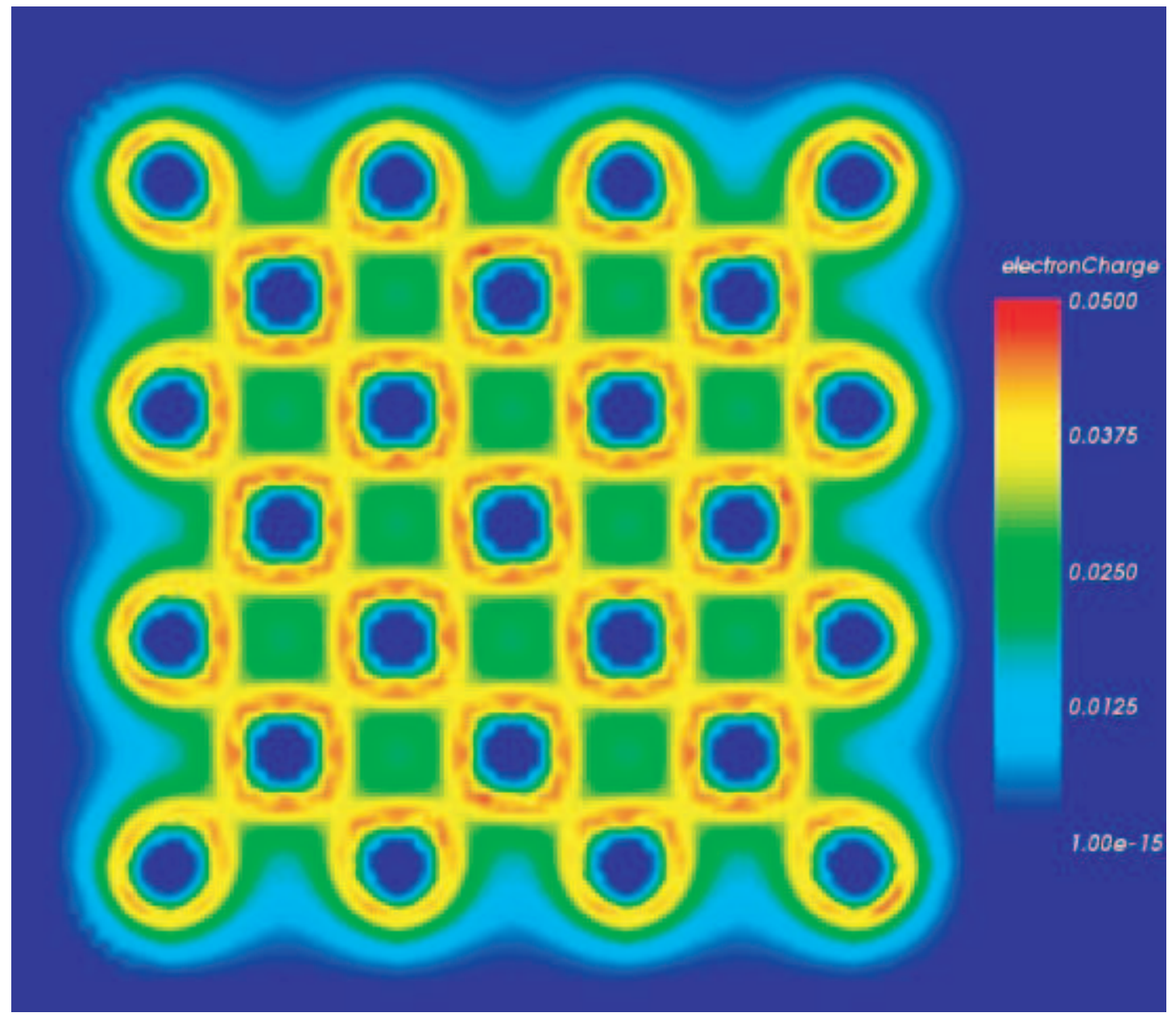

Fig. 9. Contours of electron density on the face of an aluminum cluster with $3 \times 3 \times 3$ fcc unit cells 


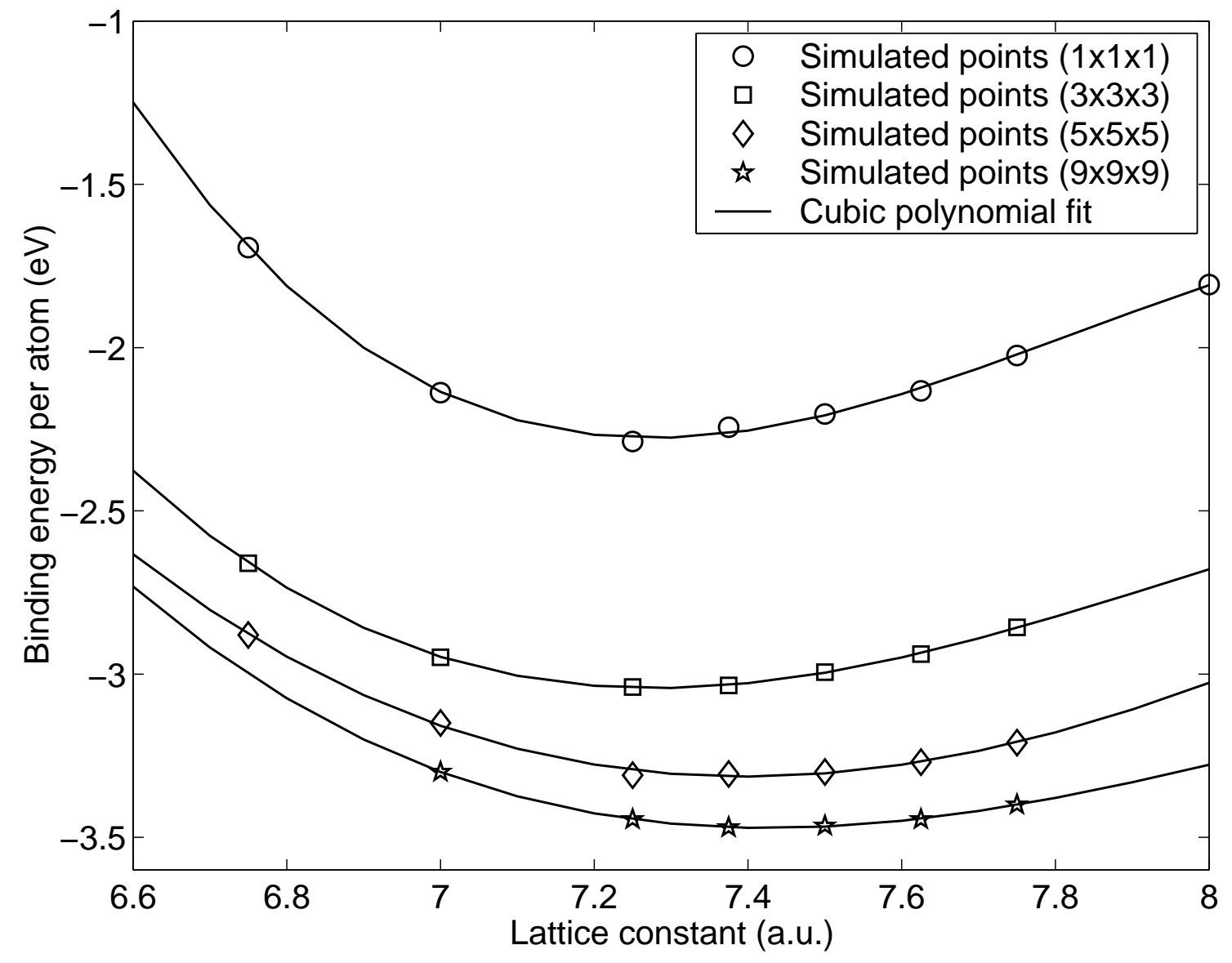

Fig. 10. Binding energy per atom as a function of lattice constant in a fcc cluster with $1 \times 1 \times 1$, $3 \times 3 \times 3,5 \times 5 \times 5$ and $9 \times 9 \times 9$ unit cells of aluminum atoms, computed using DFT-FE 


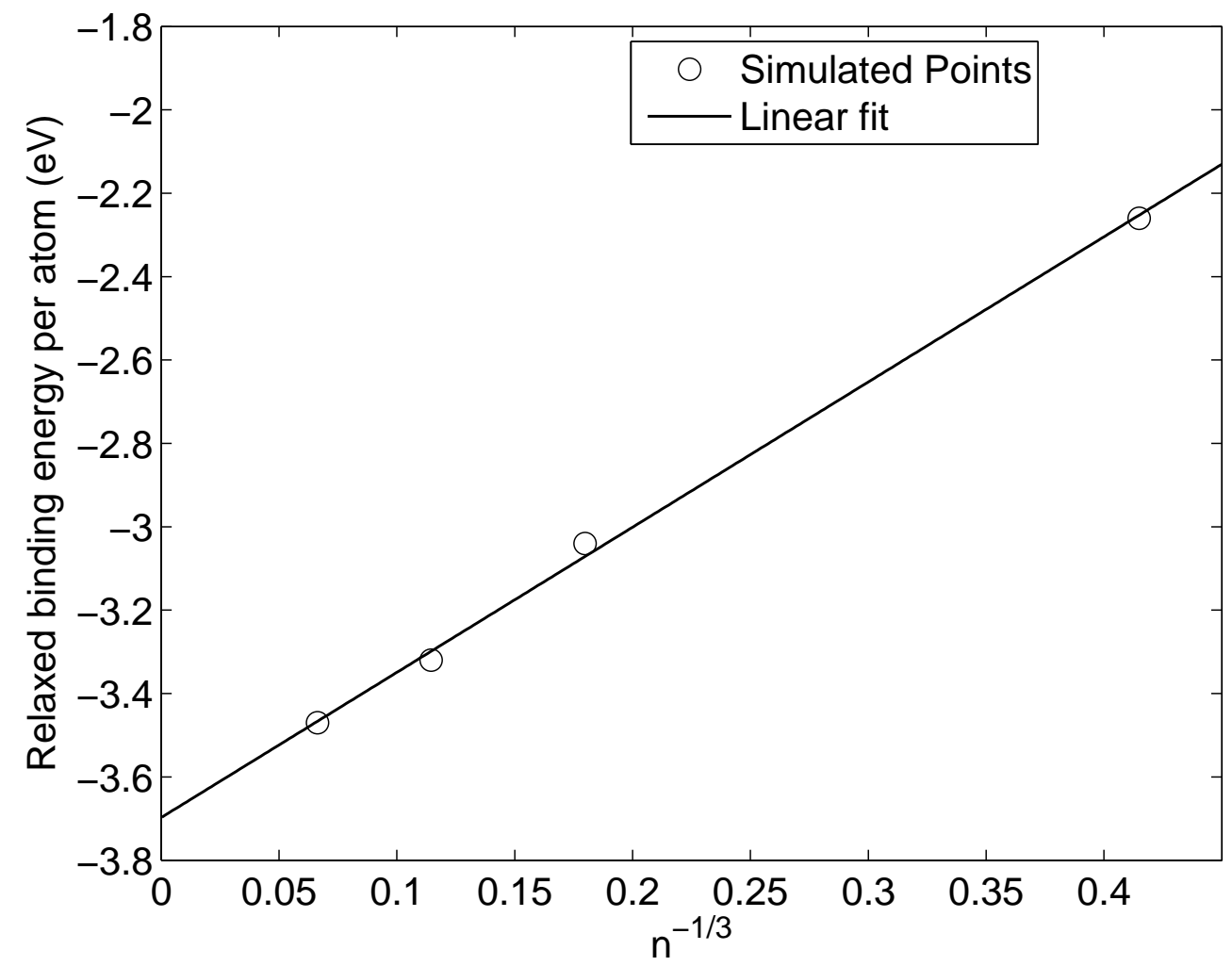

Fig. 11. Relaxed binding energies per atom of aluminum clusters against $n^{-1 / 3}$ 


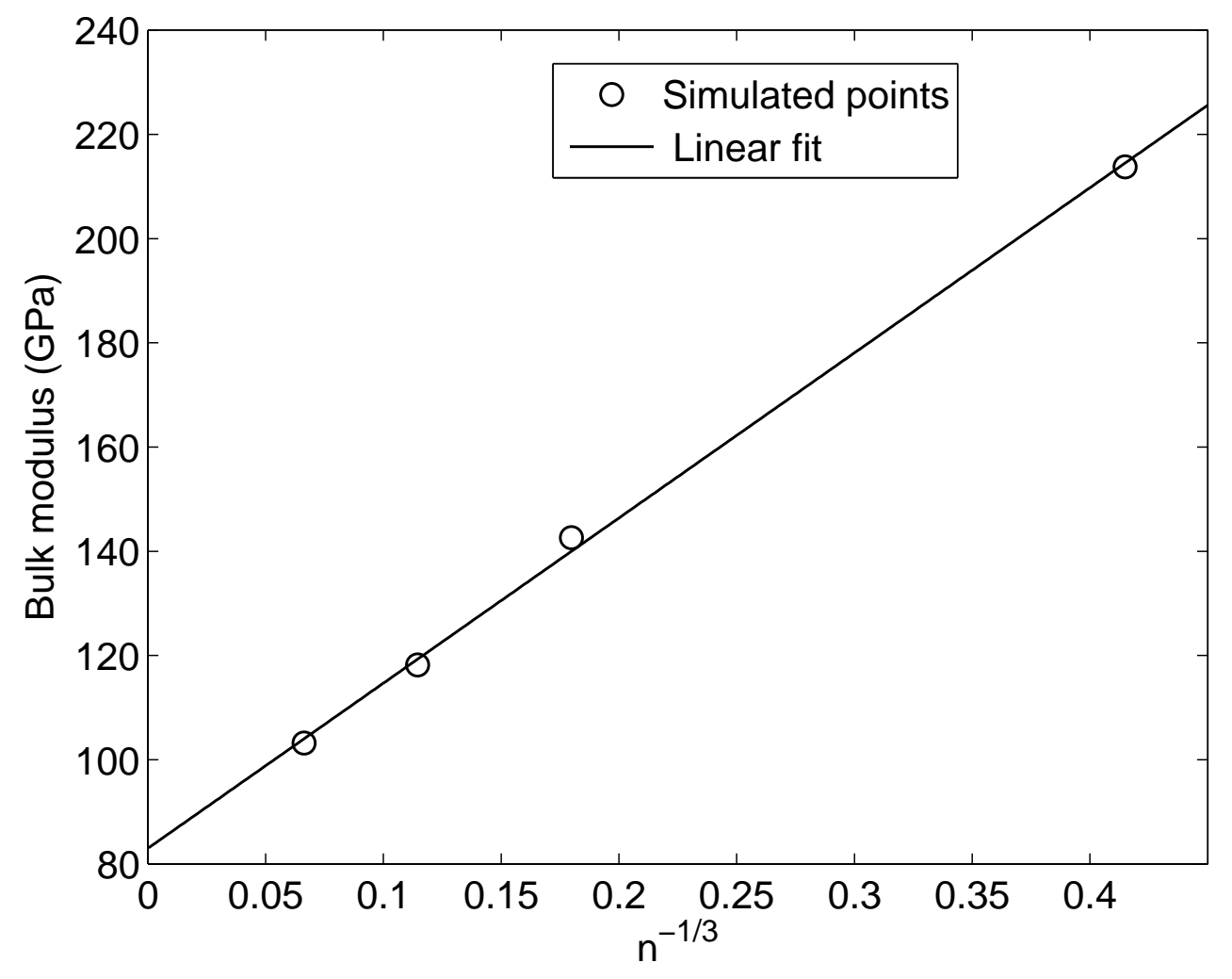

Fig. 12. Bulk modulus of aluminum clusters against $n^{-1 / 3}$ 